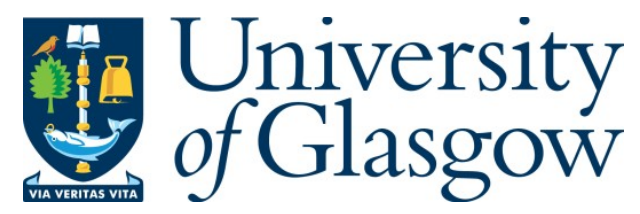

Onireti, O., Heliot, F., and Imran, M. A. (2013) On the energy efficiency-spectral efficiency trade-off of distributed MIMO systems. IEEE Transactions on Communications, 61(9), pp. 3741-3753.

There may be differences between this version and the published version. You are advised to consult the publisher's version if you wish to cite from it.

http://eprints.gla.ac.uk/136184/

Deposited on: 2 February 2017

Enlighten - Research publications by members of the University of Glasgow http://eprints.gla.ac.uk 


\title{
On the Energy Efficiency-Spectral Efficiency Trade-off of Distributed MIMO Systems
}

\author{
Oluwakayode Onireti, Member, IEEE, Fabien Héliot, Member, IEEE, \\ and Muhammad Ali Imran Senior Member, IEEE
}

\begin{abstract}
In this paper, the trade-off between energy efficiency (EE) and spectral efficiency (SE) is analyzed for both the uplink and downlink of the distributed multiple-input multiple-output (DMIMO) system over the Rayleigh fading channel while considering different types of power consumption models (PCMs). A novel tight closed-form approximation of the DMIMO EESE trade-off is presented and a detailed analysis is provided for the scenario with practical antenna configurations. Furthermore, generic and accurate low and high-SE approximations of this trade-off are derived for any number of radio access units (RAUs) in both the uplink and downlink channels. Our expressions have been utilized for assessing both the EE gain of DMIMO over co-located MIMO (CMIMO) and the incremental EE gain of DMIMO in the downlink channel. Our results reveal that DMIMO is more energy efficient than CMIMO for cell edge users in both the idealistic and realistic PCMs; whereas in terms of the incremental EE gain, connecting the user terminal to only one RAU is the most energy efficient approach when a realistic PCM is considered.
\end{abstract}

Index Terms-Energy efficiency, spectral efficiency, trade-off, distributed MIMO, Rayleigh channels.

\section{INTRODUCTION}

I NCREASING global energy demand and soaring energy related operating cost is currently steering research in communications towards the design of energy efficient networks. Up to now, the spectral efficiency (SE) metric has been the main performance indicator for designing and optimizing wireless communication networks. The SE metric measures how efficiently a limited frequency resource is utilized but fails to give insights on how efficiently the energy is consumed and, thus, this has recently led to the introduction of the energy efficiency (EE) as a performance metric. The EE of a communication network can be measured in terms of the bit-per-joule capacity [1], which is the maximum amount of bits that can be delivered by the network per joule it consumed to do so, or by using energy consumption metric such as the traditional energy-per-bit to noise spectral density [2]. Accurate evaluation of the $\mathrm{EE}$ of a network indeed requires that its power consumption must be adequately modelled. In the literature, two forms of power consumption models (PCMs) can be identified for characterizing the EE of a communication network: the idealistic PCM which only considers transmit power [1]-[5] and; the realistic PCM which accounts for the total power consumed in the network by including in addition

The authors are with the Centre for Communication Systems Research, Faculty of Engineering \& Physical Sciences, University of Surrey, Guildford GU2 7XH, UK (e-mail: F.Heliot@Surrey.ac.uk). This work has been done within joint project, supported by Huawei Tech. Co., Ltd, China. to the transmit power, the amplifier inefficiency, processing and backhauling powers, cooling loss, etc., in its model [6][10].

The Shannon information theoretic capacity for point-topoint additive white Gaussian noise (AWGN) channel has established the existence of a fundamental trade-off between EE and SE, such that an explicit expression for this AWGN EESE trade-off can be found in [1] and [2]. However, except for the AWGN channel and deterministic channel with coloured Gaussian noise, explicit expressions of the EE-SE trade-off for most other common communication channels does not yet exist. Instead, closed-form approximations (CFAs) such as Verdú low-power approximation of [2], has been extensively utilized in the literature for mathematically characterizing this trade-off [3]-[5]. In general, explicit expressions and CFAs are not only useful for swiftly assessing the performance of complicated communication systems, but they are also useful for analyzing them and getting valuable insights on how to improve them. Moreover, their importance and usage is expected to grow rapidly with the advent of future cellular networks such as self-organizing networks, where machines will rely on such mathematical formulations for keeping the system optimized at any time. We have recently proposed an approach for deriving CFAs of the EE-SE trade-off for the point-to-point multiple-input multiple-output (MIMO) over the Rayleigh fading channel in [11], [12] and for uplink of the symmetric coordinated multi-point (CoMP) system in [13]. Contrarily to the Verdú's approach, which is only accurate in the low-SE regime, our novel approach is highly accurate in any SE regime. Bearing in mind that new generations of communication networks such as Long Term evolution (LTE) and LTE-advanced (LTE-A) are designed to operate in the midhigh SE region, there is a need for developing EE-SE trade-off CFAs that are very accurate in these regimes.

The distributed multiple-input multiple-output system (DMIMO) was originally proposed to cover dead spots in indoor wireless communications [14]. However, the range of applications for DMIMO has recently been broadened due to the increasing demand for high data rate and limited power resource in wireless networks. Indeed, DMIMO can prove useful for improving the network capacity by shortening the transmission distance [15]-[17]. In the DMIMO system, the antenna units, which is usually referred to as radio access units (RAUs), are geographically distributed contrarily to the traditional colocated MIMO (CMIMO) system where the antennas are just a few wavelength apart. The capacity gain and improved power efficiency of the DMIMO system over the CMIMO 


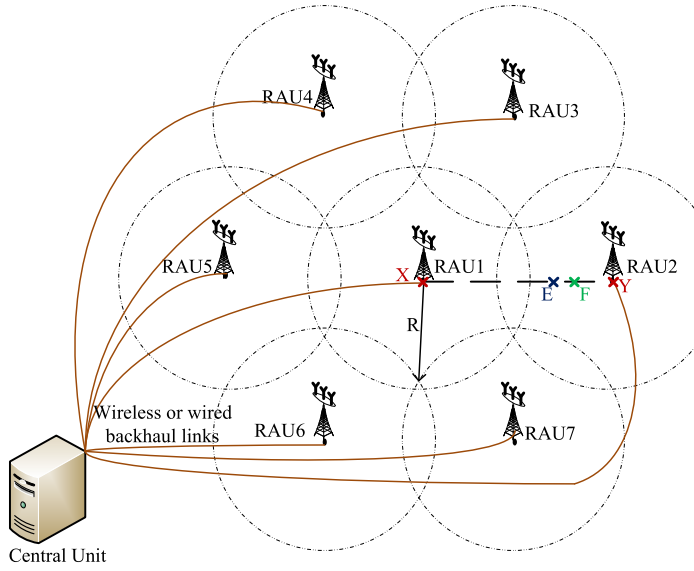

Fig. 1. Distributed MIMO system model.

scheme results from its ability to exploit both the macro and micro diversities [15]-[20]. The channel capacity of DMIMO has been extensively studied and its asymptotic closed-form expression can be found in [21], [22] while its high signalto-noise ratio (SNR) approximation has been derived in [20], [23]. As far as the uplink of the DMIMO EE-SE trade-off is concerned, its lower and upper bounds at high SNR have been obtained in [24] by using the high SNR approximation of [20], but only for some limited antenna configurations. Furthermore, an energy-efficient resource allocation for the downlink of the DMIMO system has been recently proposed in [25]. In this paper, we propose a framework for comprehensively analyzing the DMIMO system from both an EE and SE perspective by means of a tight CFA of its EE-SE trade-off. Relying on our novel approach, we go beyond [24] and propose an accurate CFA of the DMIMO EE-SE trade-off for any SE regime and wider range of antenna configurations. Section II presents the system model for the DMIMO system and reviews its capacity expression which serves as a basis for our own derivation. We then briefly present the realistic PCM of DMIMO system and formulate the actual DMIMO EE-SE trade-off expression. In Section III, we derive our CFA of the DMIMO EE-SE trade-off for any number of RAUs; we obtain this CFA for the case with a practical antenna setting by designing a parametric function and using a heuristic curve fitting method [12], [26], [27]. Numerical results show the accuracy of our approximation for several RAUs, a wide range of SE values and numerous antenna configurations. However, obtaining the CFA of the DMIMO EE-SE trade-off is too intricate for a large number of RAUs. Consequently, we derive in Section IV generic low and high-SE approximations of the DMIMO EE-SE trade-off for both the uplink and downlink scenarios. On the one hand, results show that our novel low$\mathrm{SE}$ approximation is as accurate as the approximation of [2], but it has the advantage of a simplified formulation. On the other hand, our novel high-SE approximation is very tight with the actual EE-SE trade-off results in both the mid as well as high SE regimes, and is far more accurate than the approximation in [24]. This is due to the fact that the derivation of the approximations in [24] is based on lower and upper bounds instead of an exact formulation. As an application for our novel and accurate approximations, we study in Section $\mathrm{V}$, both analytically and numerically, the effects of connecting additional RAUs to the user terminal (UT) on the system EE and how DMIMO compares with CMIMO in terms of EE, for both idealistic and realistic PCMs. Results show that some of the SE gain that is obtained by using DMIMO system can be traded-off with power to achieve EE gain over the CMIMO system. In addition, connecting the UT to the RAU, which has the strongest channel gain to the UT, is the most energy efficient approach when a realistic PCM is considered. Finally, conclusions are drawn in Section VI. A preliminary version of this work has been reported in [28]. Herein, our DMIMO EE-SE trade-off expression has been made more generic and its low and high-SE approximations have also been derived. Furthermore, a detailed analysis of the EE gain of DMIMO over CMIMO systems and incremental EE gain of DMIMO system have been conducted.

\section{System MOdEL}

\section{A. DMIMO System Model and Channel Capacity}

We consider a DMIMO communication system which consists of $M$ RAUs, each equipped with $L$ antennas and a UT equipped with $T$ antennas, as it is illustrated in Fig. 1 for the case of $M=7$. The main processing is performed at the central unit (CU) which itself is connected to the RAUs via a high-speed delay-less error-free channel such as optical fibre links. The RAUs and CU exchange signalling information, and they are assumed to be perfectly synchronized. We define the total number of transmit and receive antennas in the DMIMO system as $N_{t}$ and $N_{r}$, respectively, where $N_{t}=T$ and $N_{r}=M L$ in the uplink direction, while $N_{t}=M L$ and $N_{r}=T$ in the downlink case. As a result of the large distance separating each RAU and the UT, each corresponding channel matrix is formed of independent microscopic and macroscopic fading components. The matrices $\boldsymbol{\Omega}_{i}$ and $\mathbf{G}_{i}$ represent the deterministic distance dependent pathloss/shadowing (macroscopic fading component) and the MIMO Rayleigh fading channel (microscopic fading component), respectively, between the $i^{t h}$ RAU and the UT, where $i \in\{1, \ldots, M\}$. The channel model of the DMIMO system can then be defined as $\mathbf{H}=\boldsymbol{\Omega} \odot \mathbf{G}$, where $\mathbf{G}=\left[\mathbf{G}_{1}^{\dagger}, \mathbf{G}_{2}^{\dagger}, \ldots, \mathbf{G}_{M}^{\dagger}\right]^{\dagger},(.)^{\dagger}$ is the complex conjugate transpose, $\odot$ denotes the Hadamard product, $\mathbf{H} \in \mathbb{C}^{N_{r} \times N_{t}}, \mathbf{G} \in \mathbb{C}^{N_{r} \times N_{t}}$ and $\boldsymbol{\Omega} \in \mathbb{R}_{+}^{N_{r} \times N_{t}}$ with $R_{+}=\{x \in \mathbb{R} \mid x \geq 0\}$. Moreover, due to the multiple antennas at the UT and RAUs, the matrix $\boldsymbol{\Omega}$ is such that $\boldsymbol{\Omega}=\boldsymbol{\Lambda} \triangleq\left[\alpha_{1(D)}, \ldots \alpha_{M(D)}\right]^{\dagger} \otimes \mathbf{1}_{L \times T}$ in the uplink and $\boldsymbol{\Omega}=\boldsymbol{\Lambda}^{\dagger}$ in the downlink, where $\otimes$ denotes the Kronecker product, $\mathbf{1}_{L \times T}$ is a $L \times T$ matrix of ones. Furthermore, $\alpha_{i(D)}$ is obtained from a power-law pathloss function such as $\alpha_{i(D)}=\sqrt{L_{0}\left(1+D / D_{0}\right)^{-\eta}}$, where $L_{0}$ is the power loss at reference distance $D_{0}$ and $\eta$ is the pathloss exponent. We assume that $\mathbf{G}$ is a random matrix having i.i.d. complex circular Gaussian entries with zero-mean and unit variance.

DMIMO Channel Capacity : In the case that the channel state information (CSI) is unknown at the transmitting node and perfectly known at the receiver, equal power allocation is adopted at the transmitter. Thus, the ergodic channel capacity 
per unit bandwidth of the DMIMO system in both the uplink and downlink channels can then be expressed according to [15], [20] as

$$
\mathcal{C}=f(\gamma)=\mathbb{E}_{H}\left\{\log _{2}\left|\mathbf{I}_{N_{r}}+\frac{\gamma}{n} \mathbf{H H}^{\dagger}\right|\right\}
$$

where |.| is the determinant of a matrix, $\mathbf{I}_{N_{r}}$ is a $N_{r} \times N_{r}$ identity matrix, $\mathbb{E}$ is the expectation, $\gamma \triangleq \frac{P}{N_{0} B}$ is the average SNR, $P$ is the average transmit power per node, $B$ is the bandwidth, $N_{0}$ is the noise spectral density and $n$ is the number of transmit antenna per node. Note that $n=T$ in the uplink and $n=L$ in the downlink.

Asymptotic Approximation of the DMIMO System Capacity: It has been shown in [21], [22] that the mutual information of the DMIMO system is asymptotically equivalent to a Gaussian random variable such that both the uplink and downlink ergodic capacity per unit bandwidth can be approximated as

$$
\begin{aligned}
f(\gamma) \approx \tilde{f}(\gamma)= & \frac{n}{2 \ln (2)}\left[\kappa \sum_{i=1}^{M}\left(-1+2 \ln \left(1+\frac{d_{0} \alpha_{i}^{2}}{\rho}\right)+\frac{1}{1+d_{0} \alpha_{i}^{2} / \rho}\right)\right. \\
& \left.+\beta\left(-1+2 \ln (1+g)+\frac{1}{1+g}\right)\right](\mathrm{bit} / \mathrm{s} / \mathrm{Hz})
\end{aligned}
$$

for large values of $N_{t}$ and $N_{r}$, where $g=$ $\kappa \sum_{i=1}^{M} \alpha_{i}^{2}\left(\rho^{2}+d_{0} \alpha_{i}^{2} \rho\right)^{-1}, \rho=1 / \sqrt{\gamma}, \kappa=\frac{L}{T}$ and $\beta=1$ in the uplink while $\kappa=1$ and $\beta=\frac{T}{L}$ in the downlink. In addition, $d_{0}$ is the unique positive real root of the $(M+1)^{t h}$ degree polynomial equation

$$
P_{m}(d)=(d \rho-\beta) \prod_{i=1}^{M}\left(d+\rho v_{i}\right)+d \kappa \sum_{i=1}^{M} \prod_{\substack{k=1 \\ k \neq i}}^{M}\left(d+\rho v_{k}\right),
$$

where $v_{i}=1 / \alpha_{i}^{2}$. In order to simplify the analysis, $d_{i}$ can be defined as $d_{i}=d_{0} \alpha_{i}^{2} / \rho$ in (2) and, hence, the capacity per unit bandwidth of DMIMO given in (2) can be re-expressed as

$$
\tilde{f}(\gamma)=\frac{1}{\ln (2)}\left(S_{T}+\sum_{i=1}^{M} S_{L_{i}}\right)
$$

where $S_{T}$ and $S_{L_{i}}$ are given by

$$
\begin{aligned}
S_{T} & =\frac{T}{2}\left(-1+\frac{1}{1+g}+2 \ln (1+g)\right) \quad \text { and } \\
S_{L_{i}} & =\frac{L}{2}\left(-1+\frac{1}{1+d_{i}}+2 \ln \left(1+d_{i}\right)\right), \forall i \in\{1, \ldots, M\}
\end{aligned}
$$

respectively, for both the uplink and downlink channels.

\section{B. DMIMO Power Model and EE-SE Trade-off Formulation}

DMIMO Power Consumption Model: The EE of a communication system is closely related to its total power consumption. In a realistic DMIMO setting, power components such as the signal processing, DC-DC/AC-DC converter as well as backhaul powers and power losses from cooling, main supply as well as amplifier inefficiency must be taken into account in addition to the transmit power when evaluating the actual EE of such a system. In order to model the power consumption of DMIMO system, we assume that each RAU

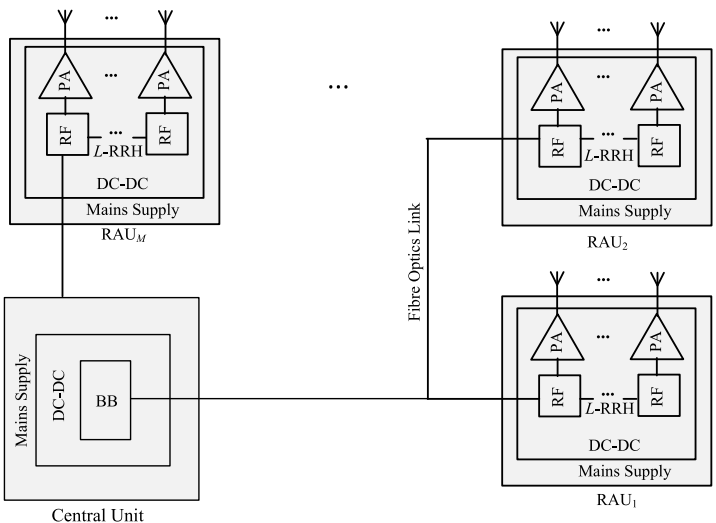

Fig. 2. Distributed MIMO power model.

is a remote radio head (RRH), such that the power amplifier (PA) and radio frequency (RF) units are mounted at the same physical location as the RAU, whereas the baseband (BB) processing unit is located at the $\mathrm{CU}$, as it is illustrated in Fig. 2. In comparison with the usual base station (BS) transceiver, a RRH transceiver does not require feeder cables such that feeder loss is mitigated; furthermore, its PAs are naturally cooled by air circulation and, hence, cooling unit is not necessary. By utilizing the realistic PCM for RRH in [8], the total consumed powers in the uplink and downlink of the DMIMO system are given by

$$
\begin{gathered}
P_{T_{u}}=\Gamma_{U T} P+T P_{c t}+M\left(L P_{0_{u}}+P_{b h}\right) \text { and }, \\
P_{T_{d}}=M\left(\Gamma_{B S} P+L P_{0_{d}}+P_{b h}\right)+T P_{c r},
\end{gathered}
$$

respectively, where $P_{c t}$ and $P_{c r}$ are the UT's transmit and receive circuit power, respectively, $\Gamma_{U T}$ quantifies the UT amplifier efficiency, and $P_{b h}$ is the backhauling induced power. Furthermore, $P_{0_{u}}$ and $P_{0_{d}}$ are the uplink and downlink power consumptions of the BS at minimum non-zero output power, and $\Gamma_{B S}$ is the slope of the load-dependent power consumption. In addition, $P \in\left[0, P_{\max }\right]$, with $P_{\max }$ being the maximum transmit power. We assume that the backhauling architecture is based on fibre optic and that all switches and interfaces are identical as in [9]. Moreover, an optical smallform factor pluggable (SFP) interface is used to transmit data from each RAU over the backhauling fibre and it has a power consumption of $c$ Watts, hence, the total backhauling induced power $P_{b h}$ per RAU can be expressed according to [9] as

$$
P_{b h}(\mathcal{C})=\frac{1}{\max _{d l}}\left(\phi p_{b}+(1-\phi) \frac{A g_{\text {switch }}(\mathcal{C})}{A g_{\max }} p_{b}\right)+p_{d l}+c,
$$

where $\max _{d l}$ is the number of interfaces per aggregation switch, $p_{d l}$ is the power consumed by one interface in the aggregation switch that is used to receive the backhauled traffic at the $\mathrm{CU}, p_{b}$ is the maximum power consumption of the switch, i.e. when all the interfaces are utilized, $\phi \in[0,1]$ represents a weighting factor. In addition, $A g_{\max }$ and $A g_{\text {switch }}$ denote the maximum and actual amounts of traffic passing through the switch, respectively, where $A g_{\text {switch }}$ is linearly dependent on the capacity per unit bandwidth of the system. 
DMIMO EE-SE Trade-off: According to [20], the bit-perJoule capacity of an energy limited wireless network is the maximum amount of bits that can be delivered per joule of consumed energy in the network, i.e. the ratio of the capacity of the system to the total consumed power, such that $\mathcal{C}_{J}=\frac{C}{P_{T}}$ in the general case, where $C=\mathcal{C} B$ is the capacity of the system in bit/s and $P_{T}$ is the total consumed power of the system. Consequently, the EE-SE trade-off for the uplink and downlink of DMIMO system can simply be expressed as

$$
\mathcal{C}_{J_{u}}=\frac{\mathcal{C}}{N_{0}}\left[\Gamma_{U T} f^{-1}(\mathcal{C})+\frac{T P_{c t}+M\left(L P_{0_{u}}+P_{b h}(\mathcal{C})\right)}{N}\right]^{-1}
$$

and

$$
\mathcal{C}_{J_{d}}=\frac{\mathcal{C}}{M N_{0}}\left[\Gamma_{B S} f^{-1}(\mathcal{C})+\frac{L P_{0_{d}}+P_{b h}(\mathcal{C})+\frac{T}{M} P_{c r}}{N}\right]^{-1},
$$

respectively, based on the realistic uplink and downlink total power consumptions given in (6) and (7). In (9) and (10), $f^{-1}: \mathcal{C} \in[0,+\infty] \mapsto \gamma \in[0,+\infty]$ is the inverse function of $f$ and $N=N_{0} B$ is the noise power. Note that in the idealistic PCM, $P_{T_{u}}=P$ and $P_{T_{d}}=M P$ such that $\mathcal{C}_{J_{u}}$ and $\mathcal{C}_{J_{d}}$ are expressed as in (9) and (10) for the uplink and downlink scenarios, respectively, but with $P_{0_{d}}=P_{0_{u}}=P_{c t}=$ $P_{c r}=P_{b h}=0$ and $\Gamma_{B S}=\Gamma_{U T}=1$.

\section{Closed-Form Approximation of the DMiMO EE-SE TRADE-OFF}

The EE-SE trade-off expressions of the DMIMO system given in (9) and (10) require the knowledge of $f^{-1}(\mathcal{C})$, however, obtaining an explicit solution of $f^{-1}(\mathcal{C})$ from the ergodic capacity expression in (1) is hardly feasible. Meanwhile, $\tilde{f}(\gamma)$ in (4) can be inverted, as it is explained in the following theorem, and since $\tilde{f}(\gamma)$ is an accurate approximation of $f(\gamma)$, $\tilde{f}^{-1}(\mathcal{C})$ is very likely to be an accurate approximation of $f^{-1}(\mathcal{C})$.

Theorem III.1: An accurate CFA of the inverse function, $f^{-1}(\mathcal{C})$, used in evaluating the EE-SE trade-off for both the uplink and downlink of the DMIMO system over the Rayleigh fading channel can be expressed as

$$
\begin{aligned}
& \tilde{f}^{-1}(\mathcal{C})= \\
& \quad \frac{-\left[1+W_{0}\left(g_{T}\left(S_{T}\right)\right)^{-1}\right]\left(\sum_{i=1}^{M}\left(\frac{-\left[1+W_{0}\left(g_{L}\left(S_{L_{i}}\right)\right)^{-1}\right]}{\Delta_{i}}-\frac{1}{\Delta_{i}}+1\right)\right)-M}{2 M\left(\kappa \sum_{i=1}^{M} \alpha_{i}^{2} x_{i}+\alpha_{1}^{2} \beta\right)},
\end{aligned}
$$

where $g_{T}\left(S_{T}\right)=-\exp \left(-\left(\frac{S_{T}}{T}+\frac{1}{2}+\ln (2)\right)\right), g_{L}\left(S_{L_{i}}\right)=$ $-\exp \left(-\left(\frac{S_{L_{i}}}{L}+\frac{1}{2}+\ln (2)\right)\right), x_{i}=\frac{W_{0}\left(g_{L}\left(S_{L_{i}}\right)\right)}{W_{0}\left(g_{L}\left(S_{L_{1}}\right)\right)}$ and $\Delta_{i}=$ $\frac{\alpha_{i}^{2}}{\alpha^{2}}$. In addition, $W_{0}(x)$ is the real branch of the Lambert function. The Lambert $W$ function is the inverse function of $f(w)=w \exp (w)$ and is such that $W(z) e^{W(z)}=z$, where $w, z \in \mathbb{C}$ [29].

Proof: See Section A of the Appendix.

Note that $S_{T}$ and $S_{L_{i}}$ in (5) are functions of $\gamma$. Thus, in order to use (11) we first need to express $S_{T}$ and $S_{L_{i}}$ as a function of $\mathcal{C}$.

We know from (4) that $\mathcal{C} \ln (2) \approx S_{T}+\sum S_{L_{i}}$, then if we could define $S_{T}-\sum S_{L_{i}}$ and $S_{L_{i}} / S_{L_{1}}, \forall i \in\{1, \ldots, M\}$, as a function of $\mathcal{C}$, we could easily express independently $S_{T}$

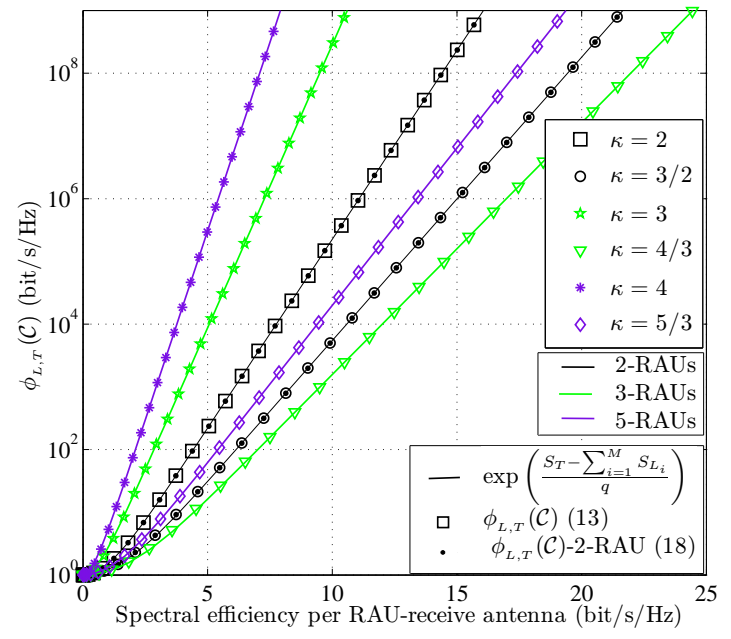

Fig. 3. Comparison of $e^{\frac{S_{T}-\sum_{i=1}^{M} S_{L_{i}}}{T}}$ with the parametric function $\phi_{L, T}(\mathcal{C})$ obtained from (13) and (18) as a function of the spectral efficiency per $R A U$-receive antenna for various number of RAUs, receive/transmit antenna ratios with $\kappa=\frac{1}{\beta}>1$ and $\Delta_{i}=10(i-1) \mathrm{dB}$.

and $S_{L_{i}}, \forall i \in\{1, \ldots, M\}$, as a function of $\mathcal{C}$ by solving a set of $M+1$ equations.

1) Approximation of $S_{T}-\sum S_{L_{i}}$ : In the following, we propose to find a parametric function $\phi_{L, T}$ that accurately approximate $S_{T}-\sum S_{L_{i}}$ by means of an heuristic curve fitting method [26] such that

$$
\phi_{L, T}(\mathcal{C}) \approx e^{\frac{S_{T}-\sum_{T} s_{L_{i}}}{T}}=\left(\frac{(1+g)^{T}}{\prod_{i=1}^{M}\left(1+d_{i}\right)^{L}}\right)^{1 / T},
$$

since it can be easily proved by direct substitution that $L\left(-1+\frac{1}{1+g}\right)-T\left(-M+\sum_{i=1}^{M} \frac{1}{1+d_{i}}\right)=0$ in (5). Note that such a method has been successfully applied in [11], [12] for defining the point-to-point MIMO EE-SE trade-off. In the heuristic curve fitting method proposed in [26], a parametric function is designed in terms of elementary functions and three independent parameters for solving a curve fitting problem. In this paper, we use this method to design the parametric function $\phi_{L, T}(\mathcal{C})$ that tightly fits $e^{\frac{S_{T}-\sum_{T} S_{L_{i}}}{T}}$ for $L>T$. We first numerically evaluated $e^{\frac{S_{T}-\sum_{T} S_{L_{i}}}{T}}$ as a function of $\mathcal{C}$ for a fixed channel gain offset, i.e. $\Delta_{i}=\alpha_{i}^{2} / \alpha_{1}^{2}, \forall i \in\{1, \ldots, M\}$, and also for various values of $M, L$ and $T$, as it is shown in Fig. 3. Similar to the MIMO case in [11], [12], $e^{\frac{S_{T}-\sum S_{L_{i}}}{T}}$ presents the feature of a logarithmic function at low $\mathcal{C}$ and linear function at high $\mathcal{C}$. The function is also monotonic and its value at $\mathcal{C}=0$ is zero. In order to define the function that best fits the curves of Fig. 3, the curve fitting method leads us to the following parametric function

$$
\phi_{L, T}(\mathcal{C})=(\cosh (\mathcal{C} \ln (2) /(T \eta)))^{\eta}
$$

which provides a satisfying approximation for $e^{\frac{S_{T}-\sum_{T} S_{L_{i}}}{T}}$, as it is shown in Fig. 3. Then, we obtain $S_{T}$ as

$$
S_{T} \approx 0.5(\mathcal{C} \ln (2)+T \eta \ln (\cosh (\mathcal{C} \ln (2) /(T \eta)))),
$$




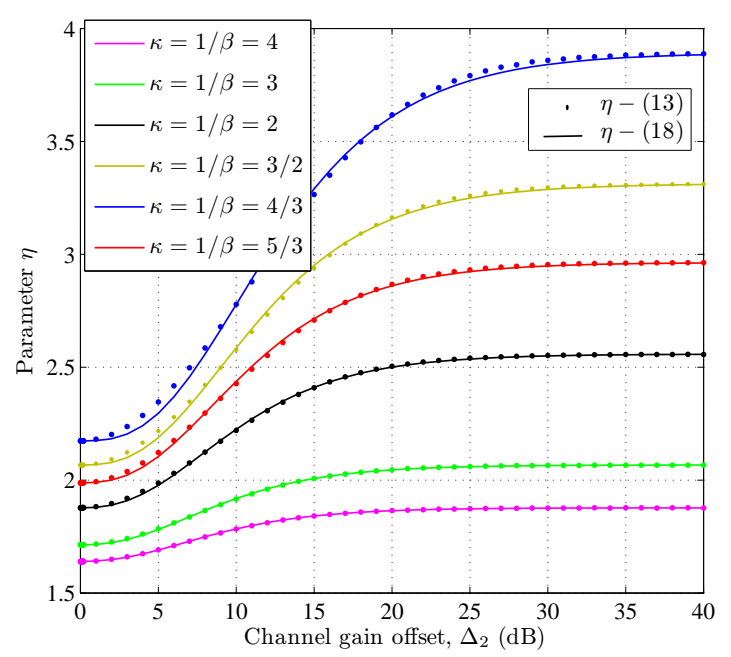

Fig. 4. Comparison of the parameter $\eta$ obtained from (13) with $\eta$ obtained via our interpolation approach of (18) as a function of the channel gain offset $\Delta_{2}$ in $\mathrm{dB}$ for the 2-RAUs scenario.

by solving equations (13) and $\mathcal{C} \ln (2) \approx S_{T}+\sum_{i=1}^{M} S_{L_{i}}$.

2) Approximation of $S_{L_{i}}$ : Furthermore, by solving equations (13) and $\mathcal{C} \ln (2) \approx S_{T}+\sum_{i=1}^{M} S_{L_{i}}$, we also obtain that

$$
\sum_{i=1}^{M} S_{L_{i}} \approx 0.5\left(\mathcal{C} \ln (2)-T \eta \ln \left(\cosh \left(\frac{\mathcal{C} \ln (2)}{T \eta}\right)\right)\right) \text {. }
$$

In our scenario of interest where $L>T$, then $S_{T} \gg S_{L_{i}}$ and, hence, it is sufficient to evaluate any $S_{L_{i}} \forall i \in\{1, \ldots, M\}$ based on its low-SE approximation. Moreover, from the proof of the DMIMO EE-SE trade-off in the low-SE regime of Proposition IV.1, we know that the ratio $\frac{S_{L_{i}}}{S_{L_{1}}}=\Delta_{i}$ at low SE. Consequently, any $S_{L_{i}}$ can be approximated as

$S_{L_{i}} \approx$

$$
\frac{\alpha_{i}^{2}}{2 \sum_{i=1}^{M} \alpha_{i}^{2}}\left(\mathcal{C} \ln (2)-T \eta \ln \left(\cosh \left(\frac{\mathcal{C} \ln (2)}{T \eta}\right)\right)\right), i \in\{1, \ldots M\} \text {. }
$$

\section{A. DMIMO System with $M=1-R A U$}

The case in which only one RAU is active, i.e. the 1-RAU DMIMO case is a very important scenario since it is equivalent to the CMIMO scenario. It can easily be shown that the inverse function, $\tilde{f}^{-1}(\mathcal{C})$, in $(11)$ simplifies into

$$
\tilde{f}^{-1}(\mathcal{C})=\frac{\left[1+\frac{1}{W_{0}\left(g_{T}\left(S_{T}\right)\right)}\right]\left[1+\frac{1}{W_{0}\left(g_{L}\left(S_{L_{1}}\right)\right)}\right]-1}{2 \alpha_{1}^{2}(\kappa+\beta)}
$$

in both the uplink and downlink channels, since $x_{1}=\Delta_{1}=1$ when only one RAU is active, i.e. when $M=1$. The functions $S_{T}$ and $S_{L_{1}}$ can be obtained from (14) and (15), respectively, where $\eta \equiv \varsigma_{2}$ in Table I. Note that (17) is equivalent to (12) of [11] which is utilized in obtaining the EE-SE trade-off closedform expression of the point-to-point MIMO over the Rayleigh fading channel.

\section{B. DMIMO System with $M=2-R A U s$}

The parameter $\eta$, varies with the ratio of the channel gain offset between the links, i.e. $\Delta_{i}$. In the case where only 2 RAUs are active, the absolute value of the $\log$ of $\Delta_{2}$ varies
TABLE II

PARAMETERS FOR THE SYSTEM AND POWER MODELS

\begin{tabular}{llrr}
\multicolumn{2}{c}{ Realistic PCM [8]-[10] } & \multicolumn{2}{c}{ System Parameters } \\
\cline { 1 - 2 } \cline { 4 - 4 } Parameter & Value & Parameter & Value \\
\hline$P_{0_{u}}$ & $24.8 \mathrm{~W}$ & $B$ & $10 \mathrm{MHz}$ \\
$P_{0_{d}}$ & $59.2 \mathrm{~W}$ & $N_{0}$ & $-169 \mathrm{dBm} / \mathrm{Hz}$ \\
$\Gamma_{B S}$ & 2.8 & $L_{0}$ & $34.5 \mathrm{~dB}$ \\
$P_{c r}$ & $0.1 \mathrm{~W}$ & $\eta$ & 3.5 \\
$P_{c t}$ & $0.1 \mathrm{~W}$ & $D_{0}$ & $1 \mathrm{~m}$ \\
$\Gamma_{U T}$ & $100 \%$ & $P_{\max }$ (Uplink) & $27 \mathrm{dBm}$ \\
$\phi$ & 0.5 & (downlink) & $46 \mathrm{dBm}$ \\
$\max _{d l}$ & 24 & Fading & Rayleigh flat fading \\
$p_{d l}$ & $1 \mathrm{~W}$ & & \\
$c$ & $1 \mathrm{~W}$ & & \\
$p_{b}$ & $300 \mathrm{~W}$ & & \\
$A g_{\max }$ & $24 \mathrm{~Gb} / \mathrm{s}$ & & \\
\hline
\end{tabular}

from 0 , i.e. the two channel gains $\alpha_{1}^{2}$ and $\alpha_{2}^{2}$ are equal, to $+\infty$, i.e. one of the links is far stronger than the other one such that $\alpha_{2}^{2} \gg \alpha_{1}^{2}$ or $\alpha_{1}^{2} \gg \alpha_{2}^{2}$, which corresponds to a $2 L \times T$ and $L \times T$ MIMO systems, respectively. Consequently, $\eta \in\left[\varsigma_{1}, \varsigma_{2}\right]$, where $\varsigma_{1}$ and $\varsigma_{2}$ are the respective values of $\eta$ for the $2 L \times T$ and $L \times T$ MIMO cases. According to Fig. 4, where $\eta$ is plotted as a function of $\Delta_{2}$ for $\Delta_{2}$ ranging from 0 to $40 \mathrm{~dB}$ and various antenna configurations, $\eta$ presents the feature of a tangent hyperbolic function, where $\eta=\varsigma_{1}$ at $\Delta_{2}=0 \mathrm{~dB}$ and $\eta$ converges to $\varsigma_{2}$ as $\Delta_{2} \rightarrow \infty$. Consequently, a tight approximation of $\eta$ can be defined by means of a curve fitting method as

$$
\eta \approx \varsigma_{1}+\left(\varsigma_{2}-\varsigma_{1}\right) \tanh \left(10 \log _{10}\left(\Delta_{2}\right) \lambda_{1}\right)^{\lambda_{2}}
$$

where the tightness of this approximation is shown in Fig. 4. We then obtain an accurate approximation of $S_{T}-\left(S_{L_{1}}+S_{L_{2}}\right)$ via $\phi_{L, T}(\mathcal{C})$ by inserting (18) into (13), as it is illustrated in Fig. 3. Note that the parameters $\varsigma_{1}, \varsigma_{2}, \lambda_{1}$ and $\lambda_{2}$ are given in Table I for some selected antenna settings. Inserting $\eta$ in (18) into (14) and (16), we easily obtain, $S_{T}, S_{L_{i}} \forall i \in\{1,2\}$ and $x_{i} \forall i \in\{1,2\}$ solely as a function of the variable $\mathcal{C}$ and for various parameters. Finally, our CFA of the EE-SE trade-off for the uplink and downlink of the 2-RAUs DMIMO system is then formulated by substituting $S_{T}, S_{L_{i}} \forall i \in\{1,2\}$ and $x_{i} \forall i \in\{1,2\}$ into $\tilde{f}^{-1}(\mathcal{C})$ in (11) and inserting $f^{-1}(\mathcal{C}) \approx$ $\tilde{f}^{-1}(\mathcal{C})$ in (9) and (10), respectively.

\section{Numerical Results and Discussions}

In order to present some numerical results, we consider the scenario where only RAU1 and RAU2 are active in the DMIMO architecture of Fig. 1 (resulting in a linear array) and the UT is assumed to be positioned at points $\mathrm{E}$ and $\mathrm{F}$, which are $0.6 R$ and $0.4 R$, respectively from RAU 2 with $R=100 \mathrm{~m}$ while the rest of the system parameters are given in Table II. In Figs. 5 and 6, we compare our CFA of the DMIMO EE-SE trade-off with the nearly-exact EE in the uplink and downlink, respectively, for an idealistic PCM and some specific values of $\kappa=\frac{1}{\beta}$, i.e. $\kappa=\{2,1.5,1.6,1.5\}$ which corresponds to the following antenna configurations $L \times T=\{2 \times 1,3 \times 2,5 \times 3,6 \times 4\}$. We can easily obtain $f(\mathcal{C})$ for a given $\mathcal{C}$ by using (1) and, hence, we can numerically obtain the nearly-exact $f^{-1}(\mathcal{C})$ and EE by using a linear search algorithm on (1) such that the target $\mathcal{C}$ differs from the actual $\mathcal{C}$ by less than $10^{-4}$ 
TABLE I

PARAMETERS $\delta_{1}, \delta_{2}, \lambda_{1}$ AND $\lambda_{2}$ VALUES FOR VARIOUS VAlUes OF $\kappa$ OR $\beta$

\begin{tabular}{|c|c|c|c|c||c|c|c|c|c||c|c|c|c|c|}
\hline$\kappa \mid 1 / \beta$ & $\varsigma_{1}$ & $\varsigma_{2}$ & $\lambda_{1}$ & $\lambda_{2}$ & $\kappa \mid 1 / \beta$ & $\varsigma_{1}$ & $\varsigma_{2}$ & $\lambda_{1}$ & $\lambda_{2}$ & $\kappa \mid 1 / \beta$ & $\varsigma_{1}$ & $\varsigma_{2}$ & $\lambda_{1}$ & $\lambda_{2}$ \\
\hline 10 & 1.517 & 1.597 & .1132 & 2.0640 & 3 & 1.713 & 2.067 & .1072 & 2.3121 & $3 / 2$ & 2.067 & 3.313 & .0932 & 2.8050 \\
\hline 9 & 1.526 & 1.616 & .1120 & 2.0619 & $8 / 3$ & 1.752 & 2.175 & .1036 & 2.2831 & $10 / 7$ & 2.109 & 3.520 & .0908 & 2.8369 \\
\hline 8 & 1.536 & 1.640 & .1080 & 1.9627 & $5 / 2$ & 1.777 & 2.243 & .1036 & 2.3447 & $7 / 5$ & 2.127 & 3.618 & .0904 & 2.8860 \\
\hline 7 & 1.551 & 1.671 & .1100 & 2.0683 & $7 / 3$ & 1.804 & 2.330 & .0996 & 2.2676 & $4 / 3$ & 2.175 & 3.893 & .0876 & 2.9851 \\
\hline 6 & 1.570 & 1.713 & .1128 & 2.1882 & $9 / 4$ & 1.820 & 2.389 & .0936 & 2.1345 & $9 / 7$ & 2.211 & 4.138 & .0856 & 3.0349 \\
\hline 5 & 1.597 & 1.777 & .1080 & 2.0855 & 2 & 1.877 & 2.558 & .1008 & 2.5063 & $5 / 4$ & 2.243 & 4.367 & .0824 & 2.9895 \\
\hline $9 / 2$ & 1.616 & 1.820 & .1100 & 2.1763 & $9 / 5$ & 1.938 & 2.769 & .0988 & 2.5974 & $6 / 5$ & 2.290 & 4.763 & .0804 & 3.1746 \\
\hline 4 & 1.640 & 1.877 & .1100 & 2.2472 & $7 / 4$ & 1.955 & 2.836 & .0980 & 2.6178 & $8 / 7$ & 2.352 & 5.432 & .0740 & 3.1797 \\
\hline $7 / 2$ & 1.671 & 1.955 & .1076 & 2.2272 & $5 / 3$ & 1.987 & 2.964 & .0964 & 2.6490 & $9 / 8$ & 2.389 & 5.696 & .0732 & 3.2949 \\
\hline $10 / 3$ & 1.683 & 1.987 & .1084 & 2.2805 & $8 / 5$ & 2.017 & 3.086 & .0944 & 2.6490 & $10 / 9$ & 2.391 & 5.947 & .0708 & 3.2154 \\
\hline
\end{tabular}



Fig. 5. Comparison of the EE-SE trade-off for the uplink of a 2-RAUs DMIMO system obtained via the nearly-exact approach and by our CFA when the UT is at position $E$ and F, based on the idealistic PCM.

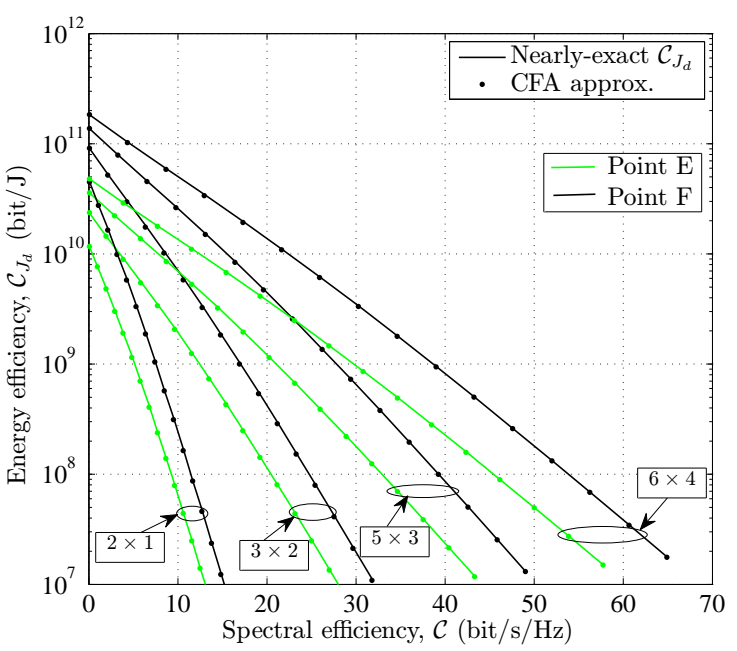

Fig. 6. Comparison of the EE-SE trade-off for the downlink of a 2RAUs DMIMO system obtained via the nearly-exact approach and by our CFA when the UT is at position $E$ and $F$, based on the idealistic PCM.
bit/s/Hz. Results clearly show the tight fitness of our CFA with the nearly-exact $\mathrm{EE}$, hence, it is a graphical illustration of the accuracy of our CFA for both the uplink and downlink channels. In addition, these results reveal that the most energy efficient point occurs at $\mathcal{C} \rightarrow 0$ when an idealistic PCM is assumed. Finally, moving the UT from E to F, i.e. closer to its serving RAU (RAU2), obviously improves the EE.

As we earlier mentioned, the parameter $\eta$ varies with the channel offset between the links when more than one RAU is active. For the case of 2-RAUs, a simple approach for obtaining $\eta$ has been defined in (18), regardless of any channel offset. However, such an approach is hardly feasible when more than 2-RAUs are active. Instead, we derive a generic and accurate approximations of the DMIMO EE-SE trade-off at low and high SE in Section IV, which we utilize in Section $\mathrm{V}$ for getting insights on the EE of DMIMO systems when $M>2$.

\section{LOW \& High-SE APPROXIMATIONS OF THE DMIMO EE-SE TRADE-OFF}

\section{A. Low-SE Approximation}

The results that have been obtained in Figs. 5 and 6, clearly indicate that the low-SE regime is the energy efficient regime in the DMIMO system when considering the idealistic PCM. According to [2, eq. (30) and (206)] the inverse function, $f^{-1}(\mathcal{C})$, used in evaluating the DMIMO EE-SE trade-off in the low-SE regime can be expressed as

$$
f^{-1}(\mathcal{C})_{\mathcal{C} \rightarrow 0}=\frac{N_{t} \mathcal{C} \ln (2)}{\mathbb{E}_{H}\left(\operatorname{tr}\left[\mathbf{H}^{\dagger} \mathbf{H}\right]\right)},
$$

where $N_{t}=T$ and $N_{t}=M L$ in the uplink and downlink cases, respectively. This formulation implies the evaluation of $\mathbb{E}_{H}\left(\operatorname{tr}\left[\mathbf{H}^{\dagger} \mathbf{H}\right]\right)$, which is tedious for large values of $N_{t}$ and $N_{r}$. A simplified version of (19) can easily be obtained by assuming that $\mathcal{C} \rightarrow 0$ in our CFA of the DMIMO trade-off in (11), as it is explained in the following proposition.

Proposition IV.1: In the low-SE regime, $\mathcal{C} \rightarrow 0$, such that (11) can be simplified and, hence, the low-SE approximation of the inverse function, $f^{-1}(\mathcal{C})$, which is utilized in characterizing the DMIMO EE-SE trade-off over the Rayleigh fading channel is simply given by

$$
\tilde{f}_{l}^{-1}(\mathcal{C})=\frac{\mathcal{C} \ln (2)}{L \beta \sum_{i=1}^{M} \alpha_{i}^{2}},
$$

where $L \beta=L$ in the uplink and $L \beta=T$ in downlink.

Proof: See Section B of the Appendix

It can be observed from (20) that the low-SE approximation of the EE-SE trade-off is independent of the number of 
transmit antennas which is in line with the results in [2], [3] for the point-to-point MIMO Rayleigh fading channel. In addition, increasing the number of receive antennas, $L \beta$, increases the $\mathrm{EE}$ as a result of an improved diversity gain.

\section{B. High-SE Approximation}

In general, high-SNR/SE approximations are of practical interest for accurately assessing the SE or EE of communication networks which operate in the mid-high SNR/SE regime. The high-SE approximation of the DMIMO EE-SE trade-off can be obtained via the high-SNR approximation of the unique real positive root of the $(M+1)^{t h}$ degree polynomial given in (3), i.e. $d_{0}$. According to [23], the formulation of the asymptotic approximation of this root is dependent on the relationship between the total number of antennas at the RAUs and the total number of antennas at the UT, i.e. $M L>T$ or $M L=T$ or $M L<T$. Using the same categorization as in [23, Lemma 1], we formulate the high-SE approximation of the DMIMO EE-SE trade-off, i.e. $\tilde{f}_{h}^{-1}(\mathcal{C})$, for both the uplink and downlink channels in the following propositions.

1) $M L>T$ :

Proposition IV.2: The high-SE approximation of the inverse function, $f^{-1}(\mathcal{C})$, used in evaluating the DMIMO EE-SE trade-off when $M L>T$ is given by

$$
\tilde{f}_{h}^{-1}(\mathcal{C})=-\frac{1}{V}\left[1+\left(2 W_{0}\left(-2^{-\left(\frac{\mathcal{C}}{T}+1\right)} e^{\left(\frac{\sum_{i=1}^{M} S_{L_{i}}^{\infty}}{T}-\frac{1}{2}\right)}\right)\right)\right],
$$

where

$$
V=\kappa\left[\sum_{i=1}^{M} \frac{\alpha_{i}^{2}\left(\kappa \sum_{k=1}^{M} \frac{\alpha_{k}^{2}}{\alpha_{1}^{2}} x_{k}^{\infty}-\beta\right)}{\kappa \sum_{k=1}^{M} \frac{\alpha_{k}^{2}}{\alpha_{1}^{2}} x_{k}^{\infty}-\beta\left(1-\frac{\alpha_{i}^{2}}{\alpha_{1}^{2}}\right)}\right]
$$

In addition, $x_{i}^{\infty}$ and $\sum_{i=1}^{M} S_{L_{i}}^{\infty}$, which are independent of $\mathcal{C}$, are given in (41) and (42), respectively.

Proof: See Section $C$ of the Appendix

2) $M L=T$ : The high-SE approximation of the inverse function, $f^{-1}(\mathcal{C})$, used in expressing the DMIMO EE-SE trade-off when $M L=T$ can be obtained by utilizing the asymptotic high-SE approximation of the unique positive real root of the $(M+1)^{t h}$ degree polynomial in (3), which can be approximated as [23]

$$
d_{0} \stackrel{\infty}{\sim} \sqrt{\sum_{i=1}^{M} \frac{1}{\alpha_{i}^{2}}}
$$

Hence, we formulate the following proposition.

Proposition IV.3: When $M L=T$, the inverse function, $f^{-1}(\mathcal{C})$, used in the DMIMO EE-SE trade-off expression can be approximated in the high SE regime as follows

$$
\tilde{f}_{h}^{-1}(\mathcal{C})=\left[\frac{(\kappa+1) \sqrt{\sum_{i=1}^{M} \frac{1}{\alpha_{i}^{2}}}}{4 \beta W_{0}\left(Q_{1}\right)}\right]^{2}
$$

where

$$
Q_{1}=-2^{-\left(\frac{\mathcal{C}}{2 T}+2\right)} e^{-\frac{M \kappa+\beta}{4 \beta}}\left(\prod_{i=1}^{M} \frac{\alpha_{i}^{2}}{\alpha_{1}^{2}}\right)^{\frac{\kappa}{2 \beta}}(\kappa+1) \sqrt{\frac{1}{\beta} \sum_{i=1}^{M} \frac{\alpha_{1}^{2}}{\alpha_{i}^{2}}} .
$$

Proof: See Section D of the Appendix
3) $M L<T$ : The asymptotic high-SE approximation of the unique positive real root of the $(M+1)^{t h}$ degree polynomial in (3) when $M L<T$ can be expressed from [23] as

$$
d_{0} \stackrel{\infty}{\sim} \sqrt{\gamma}(\beta-M \kappa)
$$

Based on the latter result, we propose the following.

Proposition IV.4: When $M L<T$, the high-SE approximation of the inverse function, $f^{-1}(\mathcal{C})$, used in the DMIMO EE-SE trade-off can be expressed as

$$
\tilde{f}_{h}^{-1}(\mathcal{C})=\frac{\sum_{i=1}^{M} \frac{1}{\alpha_{i}^{2}}}{2 M(M \kappa-\beta) W_{0}\left(Q_{2}\right)}
$$

where

$$
Q_{2}=-2^{-\frac{\mathcal{c}}{M L}} e^{-1}\left(1-\frac{M \kappa}{\beta}\right)^{-\frac{\beta}{M \kappa}}\left(\frac{\sum_{i=1}^{M} \frac{1}{\alpha_{i}^{2}}\left(\prod_{i=1}^{M} \alpha_{i}^{2}\right)^{\frac{1}{M}}}{2 M}\right)
$$

Proof: See Section E of the Appendix

It can be seen from equations (21) (23) and (26) that the high-SE approximation of the EE-SE trade-off is dependent on the capacity, $\mathcal{C}$, the number of antennas at the UT and RAU, i.e. $T$ and $L$, respectively, the channel gain between the UT and the RAU, i.e. $\alpha_{i}^{2}$ and the number of active RAU. Furthermore, by using the properties of the Lambert function, $\tilde{f}_{h}^{-1}(\mathcal{C})$ increases linearly (log scale) with linear increase in $\mathcal{C}$ when all other variables are fixed in (21), (23) and (26). Consequently, the idealistic EE decreases linearly (log scale) as $\mathcal{C}$ increases. In addition, it can be observed in (21), while $M L>T$, that increasing $T$ leads a greater improvement in the diversity gain than increasing either $M$ or $L$. Whereas, the contrary is observed in (26), where increasing either $M$ or $L$, while $M L<T$, results in a much significant improvement in the diversity gain as compared with when $T$ is increased. This improvement in diversity gain results in a reduction in $\tilde{f}_{h}^{-1}(\mathcal{C})$ and consequently an improvement in the idealistic EE, as it is depicted in Figs. 8 and 9.

\section{Numerical Results and Discussion}

In order to present some results on the low and high-SE approximations of the DMIMO EE-SE trade-off, we consider the scenario where only RAUs 1,2 and 7 are active in the DMIMO architecture of Fig. 1, i.e. $M=3$, while the UT is positioned at point $\mathrm{E}$ with $R=50 \mathrm{~m}$.

In Fig. 7, we present some numerical results on the EE of the DMIMO system at low SE for both the uplink and downlink scenarios. We compare our EE approximation at low SE in (20) with Verdú's low-SE approximation of [2], which is given in (19), for both the uplink and downlink channels in the upper and lower of Fig. 7, respectively. The results show a tight match between these two approximations. In line with the insights drawn earlier on the low-SE regime approximation, Fig. 7 show that the EE of DMIMO system is independent of the number of transmit antenna at low SE, i.e. $T$ in the uplink channel and $L$ in the downlink channel, while increasing the number of receive antennas, i.e. $L$ and $T$ in the uplink and downlink channel, respectively, improves the EE, when an idealistic PCM is considered.

In Figs. 8 and 9, we compare our high SE approximations of the DMIMO EE-SE trade-off obtained from Propositions 

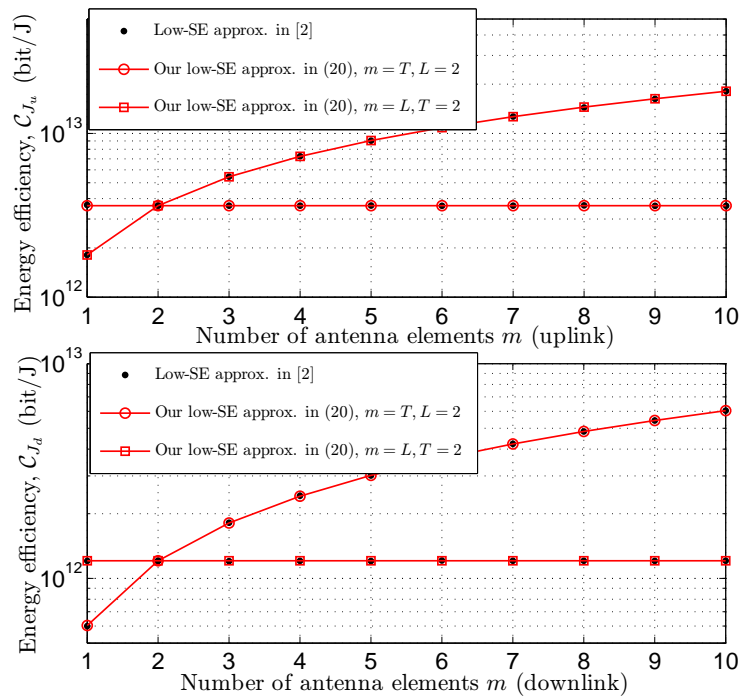

Fig. 7. Comparison of our low-SE approximation of the EE CFA for the uplink and downlink of DMIMO with the approximation in [2] when the UT is at position E, based on the idealistic PCM.

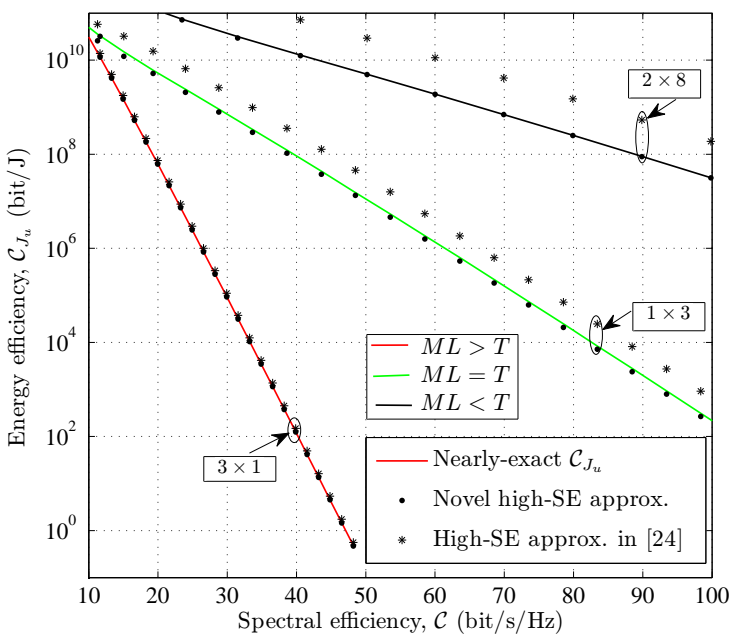

Fig. 8. Comparison of the EE-SE trade-off for the uplink of a 3-RAUs DMIMO system obtained via the nearly-exact approach with its highSE approximations when the UT is at E, based on the idealistic PCM.

IV.2, IV.3 and IV.4 for $M L>T, M L=T$ and $M L<T$, respectively, with the nearly-exact DMIMO EE-SE trade-off and the high SE approximation of [24] when considering the idealistic PCM and $M=3$. In Fig. 8, we consider the uplink of DMIMO system with the following antenna configurations $L \times$ $T=\{3 \times 1\},\{1 \times 3\}$ and $\{2 \times 8\}$ which ensure that $M L>T$, $M L=T$ and $M L<T$, respectively, since $M=3$. Whereas in Fig. 9, we consider the downlink of DMIMO system and utilize the antenna configurations $L \times T=\{1 \times 1,2 \times 2,2 \times$ $4,4 \times 2\},\{1 \times 3,2 \times 6\}$ and $\{1 \times 20,2 \times 10,2 \times 20\}$ which ensure that $M L>T, M L=T$ and $M L<T$, respectively. Note that the high-SE approximation of [24] has only been plotted for the uplink, since it is by design only valid for the uplink. The results in Fig. 8 indicates that even for the uplink scenario this

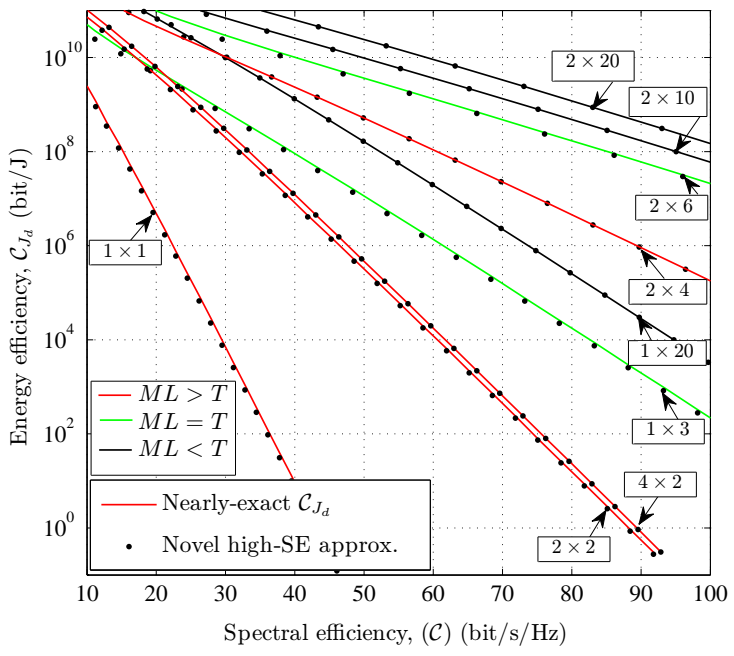

Fig. 9. Comparison of the EE-SE trade-off for the downlink of a 3-RAUs DMIMO system obtained via the nearly-exact approach with its high-SE approximations when the UT is at E, based on the idealistic PCM.

method is not very accurate, especially when $T>L$. On the contrary, our high-SE approximations are valid for both uplink and downlink and the results in Figs. 8 and 9 both indicate their great accuracy for any antenna settings, since they tightly match the nearly-exact EE results in any case. Moreover, not only our high-SE approximations are very accurate at high SE, but they are also accurate at mid-SE, i.e. for $\mathcal{C}>10 \mathrm{bit} / \mathrm{s} / \mathrm{Hz}$ in Figs. 8 and 9.

In Fig 9 we also investigate the impact of increasing the number of antennas elements at the UT, i.e. $T$, and each RAU, i.e. $L$, on the idealistic EE. For the case where $M L>T$, i.e. $L \times T=\{2 \times 2,4 \times 2,2 \times 4\}$, increasing $L$, has a less significant impact on improving the idealistic EE as compared with increasing $T$. As it can be seen in (21), $T$ is dividing $\mathcal{C}$, such that it directly affects the diversity and modify the slope of the trade-off curves, as it is clearly depicted in Fig. 9. Whereas $L$ acts as an EE multiplicative gain, since curves with different $L$ values are parallel to each other's. Note that, the contrary is observed for the case where $M L<T$, i.e. $L \times T=\{1 \times 20,2 \times 10,2 \times 20\}$. These results are in line with the insights previously drawn on equations (21) and (26).

In Fig. 10, we further demonstrate the accuracy of our high SE approximation for various number of active RAUs and a variable UT position. We plot the idealistic EE against the normalized distance (0: UT collocated at RAU1 and 1: UT collocated at RAU2) while 3 sets of RAUs are active and the UT is moving from point $\mathrm{X}$ to $\mathrm{Y}$ in the DMIMO architecture of Fig. 1, for the antenna configurations $L \times T=\{2 \times 1,2 \times 2\}$ and $\mathcal{C}=20,30 \mathrm{bit} / \mathrm{s} / \mathrm{Hz}$. The RAUs set are: 2-RAUs DMIMO with RAUs 1 and 2 active, 3-RAUs DMIMO with RAUs 1, 2 and 7 active, and 5-RAUs DMIMO with RAUs 1, 2, 5, 6 and 7 active.

\section{ENERGY EFFICIENCY ANALYSIS OF DMIMO SYSTEM}

As an application for our CFAs of Section III and IV, we study in this Section the impact of increasing the number 


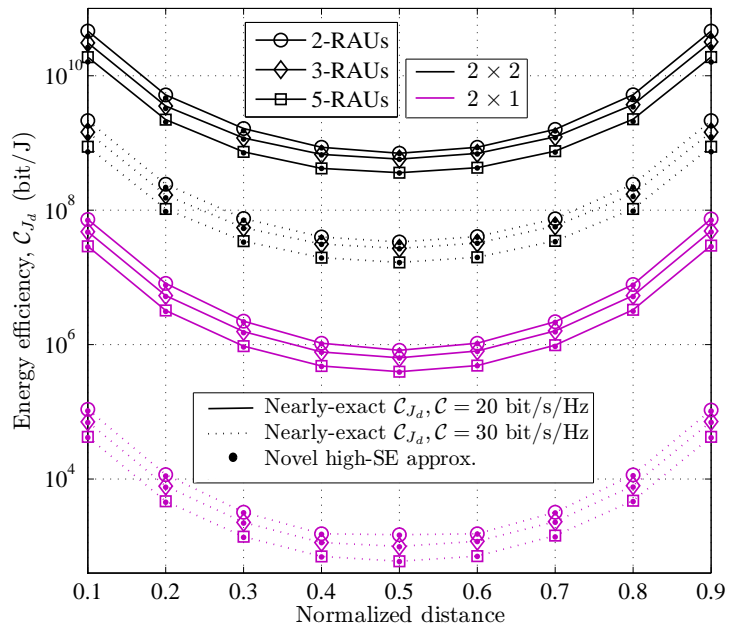

Fig. 10. Comparison of the EE-SE trade-off for the downlink of the (2-RAUs, 3-RAUs and 5-RAUs) DMIMO systems obtained via the nearly-exact approach with its high-SE when the UT is moving from point $X$ to $Y$, based on the idealistic PCM.

of RAUs on the DMIMO EE, i.e. capacity improvement, additional processing as well as power consumption, and compare the EE performance of DMIMO with CMIMO, for the downlink scenario and both idealistic as well as realistic PCMs. In this regards, we derive the incremental EE gain and EE gain of DMIMO over CMIMO in the two following subsections, respectively.

\section{A. Incremental Energy Efficiency Gain of DMIMO System}

We define here the incremental EE gain of DMIMO as the variation of EE when an additional RAU is connected to the UT. Note that the same type of analysis has been undertaken in [17] but to quantify the SE instead of the EE variations. Contrary to [17], we assume a target SE, i.e. a fixed channel capacity, and evaluate how the total power consumption is affected when extra RAUs are added. We consider as in [17] that the RAU connection order to the UT is from the closest RAU to the furthest one and define the incremental EE gain according to (10) as

$$
I G_{E}=\frac{M}{M+1}\left(\frac{\Gamma_{B S} f_{M}^{-1}(\mathcal{C})+\left(\bar{P}_{0} / N\right)}{\Gamma_{B S} f_{M+1}^{-1}(\mathcal{C})+\left(\bar{P}_{0} / N\right)}\right)
$$

where $\bar{P}_{0} \approx p P_{0_{d}}+P_{b h}(\mathcal{C})$ since $\mathrm{UT}$ receive circuit power, $P_{c r}$, is negligible. In addition, $f_{M}^{-1}(\mathcal{C})$ and $f_{M+1}^{-1}(\mathcal{C})$ are approximated in (11) for the initial DMIMO system and when an additional RAU is connected to the UT, respectively. Since obtaining the exact closed-form expressions for $f_{M}^{-1}$ and $f_{M+1}^{-1}$ is not straightforward when the number of RAUs is greater than two, we utilize their low and high-SE approximations given in Propositions IV.1 and IV.2-IV.4, respectively. Consequently, the idealistic incremental EE gains of the DMIMO system (when considering the idealistic PCM) in the low and
high-SE regimes can be approximated as

$$
\begin{aligned}
& I G_{E, I d}^{0} \approx \frac{M}{M+1} \frac{\sum_{i=1}^{M+1} \alpha_{i}^{2}}{\sum_{i=1}^{M} \alpha_{i}^{2}} \text { and } \\
& I G_{E, I d}^{\infty} \approx \frac{M}{M+1}\left(\frac{\tilde{f}_{h, M}^{-1}(\mathcal{C})}{\tilde{f}_{h, M+1}^{-1}(\mathcal{C})}\right),
\end{aligned}
$$

respectively, when $\bar{P}_{0}=0$ and $\Gamma_{B S}=1$ while $\tilde{f}_{h, M}^{-1}(\mathcal{C})$ and $\tilde{f}_{h, M+1}^{-1}(\mathcal{C})$ are the high-SE approximation of $f_{M}^{-1}(\mathcal{C})$ and $f_{M+1}^{-1}(\mathcal{C})$. Notice that in the symmetric channel case, i.e. all $\alpha_{i}^{2}$ are equal, $I G_{E, I d}^{0} \approx 1$ in (29). Furthermore, the maximum value of $I G_{E, I d}^{0}$ occurs when the links are symmetrical, hence, no EE gain can be achieved by having additional RAUs in the low-SE regime. By using (29), the realistic incremental EE gains of the DMIMO system (when considering the realistic PCM) in the low and high-SE regimes can be expressed as

$I G_{E, R e}^{0} \approx M\left(\frac{\Gamma_{B S} \tilde{f}_{l, M}^{-1}(\mathcal{C})+\left(\bar{P}_{0} / N\right)}{M \Gamma_{B S}\left(\tilde{f}_{l, M}^{-1}(\mathcal{C}) / I G_{E, I d}^{0}\right)+\left(\bar{P}_{0} / N\right)(M+1)}\right) \quad$ and

$I G_{E, R e}^{\infty} \approx M\left(\frac{\Gamma_{B S} \tilde{f}_{h, M}^{-1}(\mathcal{C})+\left(\bar{P}_{0} / N\right)}{M \Gamma_{B S}\left(\tilde{f}_{h, M}^{-1}(\mathcal{C}) / I G_{E, I d}^{\infty}\right)+\left(\bar{P}_{0} / N\right)(M+1)}\right)$,

respectively, where $\tilde{f}_{l, M}^{-1}(\mathcal{C})$ is given in (20).

\section{B. EE Gain of DMIMO System Over Co-located MIMO (CMIMO) System}

The power efficiency gain of the DMIMO system over CMIMO system where all the antenna elements are separated by a few wavelengths has been analyzed in [17] while considering the idealistic PCM. In order to evaluate how the DMIMO system compares with the CMIMO system in terms of EE over the downlink channel, we express the EE gain of DMIMO over CMIMO according to (10) and [8] as

$$
G_{E}=G_{I d, S E} \frac{\Gamma_{B S} f_{1}^{-1}\left(\mathcal{C}_{C}\right)+\left(M L P_{0_{\mathrm{d}}} / N\right)}{M\left(\Gamma_{B S} f_{M}^{-1}(\mathcal{C})+\bar{P}_{0} / N\right)}
$$

where $G_{I d, S E}=\frac{\mathcal{C}}{\mathcal{C}_{C}}$ is the idealistic SE gain, $\mathcal{C}_{C}$ and $\mathcal{C}$ are the capacities of the CMIMO and DMIMO systems, respectively, and $f_{1}^{-1}\left(\mathcal{C}_{C}\right)$ and $f_{M}^{-1}(\mathcal{C})$ are approximated in (17) and (11), respectively. From a PCM perspective, we consider that CMIMO utilizes a RRH. This EE gain can result from DMIMO transmit power reduction capability when both systems are required to achieve the same SE, i.e. $\mathcal{C}=\mathcal{C}_{C}$. The idealistic EE gain due to power reduction, which is denoted by $G_{I d, P R}$, is then simply $G_{I d, P R}=\frac{f_{1}^{-1}\left(\mathcal{C}_{C}\right)}{M f_{M}^{-1}\left(\mathcal{C}_{C}\right)}$ since $G_{I d, S E}=1$, while its realistic value, i.e. $G_{R e, P R}$, is simply a ratio of the total consumed power in the two systems as observed in (31).

The EE gain of DMIMO over CMIMO can also be approached via its SE improvement capability when a fixed total transmit power is assumed for both systems, i.e. $M f_{M}^{-1}(\mathcal{C})=$ $f_{1}^{-1}\left(\mathcal{C}_{C}\right)$. Hence, this EE gain is simply equivalent to the SE gain, i.e. $G_{I d, S E}$ in the idealistic setting. Note that the DMIMO system incorporates an additional backhauling induced power in comparison with the CMIMO system, hence the realistic EE gain as a result of its $\mathrm{SE}$ improvement capability, denoted as $G_{R e, S E}$, is always lower than $G_{I d, S E}$, as it can be seen in Fig. 13 . 


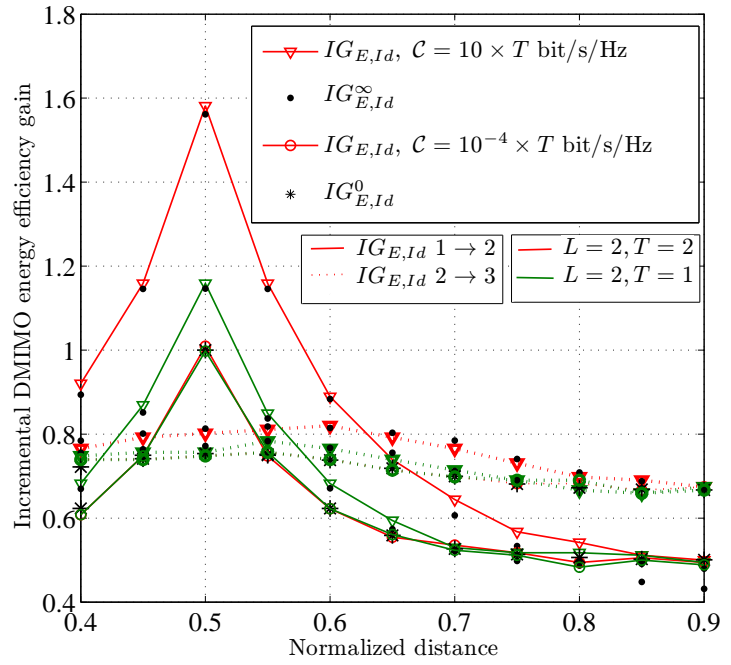

Fig. 11. Incremental EE gain of DMIMO when the UT is moving from point $X$ to $Y$, based on the idealistic PCM.

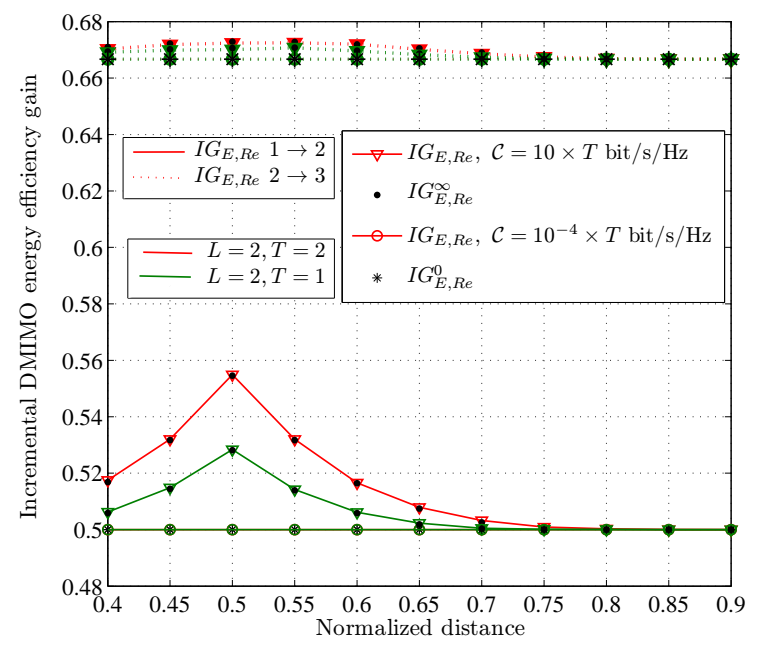

Fig. 12. Incremental EE gain of DMIMO when the UT is moving from point $X$ to $Y$, based on a realistic PCM.

\section{Numerical Results and Discussions}

In order to understand the EE behaviour of DMIMO system, we present some results on both the incremental EE gain of DMIMO and EE gain of DMIMO over CMIMO for both the idealistic and realistic PCMs over the downlink channel. We consider the DMIMO architecture of Fig. 1 with $R=100 \mathrm{~m}$ and where the UT is moving from point $\mathrm{X}$ to $\mathrm{Y}$, and rely on the realistic power consumption parameters given in Table II for plotting our results. In Figs. 11 and 12, we evaluate the idealistic and realistic incremental EE gains of DMIMO, respectively, as a function of the normalized distance ( 0 : UT collocated at RAU1 and 1: UT collocated at RAU2), for the antenna configurations $L \times T=\{2 \times 1,2 \times 2\}$, $\mathcal{C} / T=10^{-4}$ and $10 \mathrm{bit} / \mathrm{s} / \mathrm{Hz}$. We consider that only RAUs 1,2 and 7 can be active and that the connection order to the

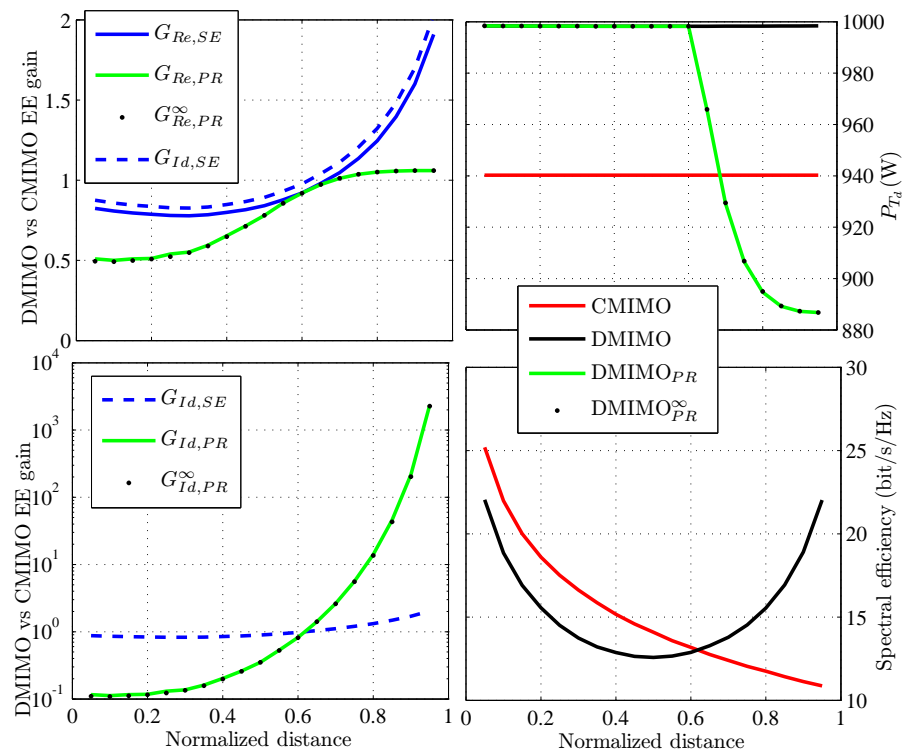

Fig. 13. EE gain of DMIMO over CMIMO when the UT is moving from point $\mathrm{X}$ to $\mathrm{Y}$, based on both the idealistic and realistic PCMs.

RAU is from the closest RAU to the furthest one. We can obtain $f_{M}^{-1}(\mathcal{C})$ by using the numerical search approach and its low and high-SE approximations from Propositions IV.1 and IV.2-IV.4, respectively. The result in Fig. 11 confirms that at low SE, $I G_{E, I d}^{0}=1$ for the symmetric case, when the number of RAUs connected to the UT increases from 1 to 2. Moreover, the incremental EE gain is independent of the number of antennas at the UT at low-SE, which is in line with the insight previously drawn. Furthermore, increasing the number of RAUs connected to the UT from one to two is only beneficial in terms of EE when the average channel gains are fairly equal and when considering the idealistic PCM. Whereas, connecting a third RAU is not beneficial in terms of EE when compared to the two RAUs scenario due to the reduced impact of the macro diversity.

In Fig. 12, we consider that the maximum transmit power per RAU does not exceed $43 \mathrm{dBm}$. The result clearly indicates that when considering the realistic PCM, no EE gain is achieved by using an additional RAU in the system. Indeed, the RAU connection order entails that the closest RAU is connected to the UT, hence the reduction in transmit power as a result of the additional RAU is minimal except when the channel gains of the two links are fairly equal. However, since other power consumptions increase linearly as the number of RAUs increase and since this increase cannot be compensated by transmit power reduction, hence a loss in EE occurs. It can also be observed that the incremental $\mathrm{EE}$ is nearly constant with the user positioning at very low SE. This is due to the fact that $P \rightarrow 0$ as $\mathcal{C} \rightarrow 0$, and that the part of the backhauling power depending on the traffic also tends to zero. Consequently, $I G_{E, R e}^{0} \simeq \frac{M}{M+1}$ as $\mathcal{C} \rightarrow 0$.

Figure 13 compares the DMIMO with CMIMO system in terms of EE for various normalized UT positions and for the antenna configuration $L \times T=\{2 \times 1\}$. We consider that all RAUs are active in the DMIMO system, i.e. $M=7$, and that all RAUs are co-located at RAU 1 in the CMIMO case. In 
the lower-right graph, we plot the SE of both the DMIMO and CMIMO systems when the total transmit power is set to $46 \mathrm{dBm}$. As it is expected, CMIMO system has a higher SE than DMIMO when the UT is within its range, since the CMIMO has $M L$ collocated antennas giving micro-diversity gain. Whereas, in the DMIMO system, each distributed RAU is equipped with $L$ antennas and the combination of macro and micro-diversity gains results in a higher SE when the UT is close to the cell edge (RAU 2). In the upper-left graph, we plot the idealistic and realistic EE gains of DMIMO system over CMIMO system i.e. $G_{I d, S E}$ and $G_{R e, S E}$, which are obtained from (31). As it can be observed, the SE improvement capability of DMIMO when the UT is in close proximity with RAU 2, results into EE gain. Furthermore, in line with our analysis, the idealistic EE gain as a result of the SE improvement ability of DMIMO, i.e. $G_{I d, S E}$ is always greater than the realistic EE gain, i.e. $G_{R e, S E}$. In order to demonstrate the EE gain of DMIMO over CMIMO as a result of power reduction, we utilize our high-SE approximation from Proposition IV.2-IV.4 to plot this EE gain for both the idealistic and realistic PCMs, i.e. $G_{I d, P R}^{\infty}$ and $G_{R e, P R}^{\infty}$, respectively, when the total transmit power of the CMIMO is fixed to $46 \mathrm{dBm}$ and the DMIMO system achieves the same SE as the CMIMO system. We also plot the EE gains, $G_{I d, P R}$ and $G_{R e, P R}$ based on a numerical search approach to further demonstrate the accuracy of our high-SE approximations. Hereafter, we plot in the upper-right graph, the total power consumption of the CMIMO and DMIMO systems when the total transmit power is fixed at $46 \mathrm{dBm}$. We also plot the total power consumption of the DMIMO system as a result of the EE gains $G_{R e, P R}$ and $G_{R e, P R}^{\infty}$ i.e. $\mathrm{DMIMO}_{P R}$ and $\mathrm{DMIMO}_{P R}^{\infty}$, respectively. The results indicate that a reduction in the total power consumption in the DMIMO system can be achieved by sacrificing the SE gain of DMIMO while transmitting at a lower power for cell edge users.

\section{CONCLUSION}

In this paper, an accurate CFA of the EE-SE trade-off for both the uplink and downlink of the DMIMO system over a Rayleigh fading channel has been derived by considering both an idealistic and realistic PCMs. In addition, simplified tight approximations of this trade-off have also been derived at low and high SE for any number of RAUs and antennas. We first proposed a formal proof of our approach for obtaining the generic CFA of the EE-SE trade-off for the DMIMO system. We then obtained this CFA by designing a parametric function and using a heuristic curve fitting method. The accuracy of our CFA has been shown graphically for various practical antenna configurations and a wide range of SE values. Since obtaining the CFA of the DMIMO EE-SE trade-off is more intricate for a large number of RAUs, we have resorted to low and highSE approximations of the EE-SE trade-off for the generic $M$ RAUs DMIMO scenario. Our approximations have proved to be very accurate, far more accurate than the existing ones for the high SE case and as accurate as the one in the literature for the low SE regime but with a simplified formulation.

The EE gain of DMIMO over CMIMO and the incremental EE gain of DMIMO in the downlink channel has been inves- tigated by using our low and high-SE approximations for both the idealistic and realistic PCMs. The realistic incremental EE gain indicated that the optimal approach in terms of EE is to connect the UT to the RAU with the strongest channel gain. In terms of the EE gain of DMIMO over CMIMO, DMIMO is more energy efficient than CMIMO for cell edge UTs in both PCMs. Furthermore, the SE gain that is obtained by using DMIMO system can be traded-off with power to achieve EE gain over the CMIMO system.

Note that the DMIMO system model presented in this paper is based on the single cell scenario with an orthogonal multiple access scheme implemented within the cell and is therefore noise limited. Since interference is a key limiting factor in cellular communication, hence the CFA of the EE-SE trade-off for the interference limited DMIMO deserves much attention in future study.

\section{APPENDIX}

\section{A. Proof of Theorem III.1}

Let $u_{i}=\frac{1}{1+d_{0} \alpha_{i}^{2} / \rho}=\frac{1}{1+d_{0} \alpha_{i}^{2} \sqrt{\gamma}}$ in (2), hence $g \triangleq$ $\kappa \sum_{i=1}^{M} \alpha_{i}^{2}\left(\rho^{2}+d_{0} \alpha_{i}^{2} \rho\right)^{-1}=\kappa \gamma \sum_{i=1}^{M} \alpha_{i}^{2} u_{i}$ and the $(M+$ $1)^{t h}$ degree polynomial equation in (3) is equivalent to

$$
\frac{d_{0}}{\beta \sqrt{\gamma}}=\frac{1}{1+g}
$$

Furthermore, $\frac{d_{0}}{\beta \sqrt{\gamma}}$ can be expressed as $\frac{d_{0}}{\beta \sqrt{\gamma}}=$ $\frac{-A+\sqrt{A^{2}+4 \alpha_{1}^{2} \gamma \beta}}{2 \alpha_{1}^{2} \gamma \beta}$, by substituting $u_{1}$ into $g$, where $A=\kappa \gamma \sum_{i=1}^{M} \alpha_{i}^{2} x_{i}+1-\alpha_{1}^{2} \gamma \beta$ and $x_{i}=\frac{u_{i}}{u_{1}}$. Moreover, $d_{i} \triangleq d_{0} \alpha_{i}^{2} \sqrt{\gamma}=\Delta_{i}\left(\frac{-A+\sqrt{A^{2}+4 \alpha_{1}^{2} \gamma \beta}}{2}\right)$ and $g$ can easily be expressed from (32) as $g \triangleq \frac{\beta \sqrt{\gamma}}{d_{0}}-1$, which is equivalent to $g=-1+\frac{A+\sqrt{A^{2}+4 \alpha_{1}^{2} \gamma \beta}}{2}$. Consequently, by defining $\bar{g}$ and $\bar{d}_{i}$ as $\bar{g}=2 g+1$ and $\bar{d}_{i}=2 d_{i}+1$, hence, we have

$$
\begin{aligned}
\bar{g} & =A+\sqrt{A^{2}+4 \alpha_{1}^{2} \gamma \beta}-1 \text { and } \\
\frac{\bar{d}_{i}}{\Delta_{i}}-\frac{1}{\Delta_{i}}+1 & =-A+\sqrt{A^{2}+4 \alpha_{1}^{2} \gamma \beta}+1 .
\end{aligned}
$$

Furthermore, by substituting the value of $A=\kappa \gamma \sum_{i=1}^{M} \alpha_{i}^{2} x_{i}+$ $1-\alpha_{1}^{2} \gamma \beta$ into (33), it can be easily shown that

$$
\bar{g} \sum_{i=1}^{M}\left(\frac{\bar{d}_{i}}{\Delta_{i}}-\frac{1}{\Delta_{i}}+1\right)=M\left(\gamma\left(2 \kappa \sum_{i=1}^{M} \alpha_{i}^{2} x_{i}+2 \alpha_{1}^{2} \beta\right)+1\right)
$$

and, hence, the CFA of the inverse function, $f^{-1}(\mathcal{C})$, used in the DMIMO EE-SE trade-off formulation can be expressed as

$$
\gamma \approx \tilde{f}^{-1}(\mathcal{C})=\frac{\bar{g} \sum_{i=1}^{M}\left(\frac{\bar{d}_{i}}{\Delta_{i}}-\frac{1}{\Delta_{i}}+1\right)-M}{2 M\left(\kappa \sum_{i=1}^{M} \alpha_{i}^{2} x_{i}+\alpha_{1}^{2} \beta\right)} .
$$

The first equation in (5) is equivalent to $-\left(\frac{S_{T}}{T}+\frac{1}{2}+\ln (2)\right)=-[1+\bar{g}]^{-1}+\ln (1+\bar{g})$ hence

$$
g_{T}\left(S_{T}\right)=-\frac{1}{1+\bar{g}} \exp \left(-\frac{1}{1+\bar{g}}\right)
$$

which can be re-formulated by using the Lambert $W$ function [29] as 


$$
\begin{aligned}
-\frac{1}{1+\bar{g}} & =W_{0}\left(g_{T}\left(S_{T}\right)\right) \\
\bar{g} & =-\left[1+\frac{1}{W_{0}\left(g_{T}\left(S_{T}\right)\right)}\right],
\end{aligned}
$$

where $g_{T}\left(S_{T}\right)=-\exp \left(-\left(\frac{S_{T}}{T}+\frac{1}{2}+\ln (2)\right)\right)$. Similarly, $\bar{d}_{i}=$ $-\left[1+\frac{1}{W_{0}\left(g_{L}\left(S_{L_{i}}\right)\right)}\right]$ and, moreover, $x_{i}$ can be easily expressed as $x_{i}=\frac{W_{0}\left(g_{L}\left(S_{L_{i}}\right)\right)}{W_{0}\left(g_{L}\left(S_{L_{1}}\right)\right)}, \forall i \in\{1, \ldots, M\}$, by noting that $u_{i}=$ $2 /\left(1+\bar{d}_{i}\right)$. Finally, (11) is obtained by inserting $\bar{g}$ in (37) and $\bar{d}_{i}$ into (35).

\section{B. Proof of Proposition IV.1}

In the low-SE regime, $\mathcal{C} \rightarrow 0$ and, hence, $S_{T}=\sum_{i=1}^{M} S_{L_{i}}$. Moreover, by applying the following approximations of the functions $\ln (1+x) \stackrel{0}{\sim} x$ and $\frac{1}{1+x} \stackrel{0}{\sim} 1-x$ to (5), the ratio $\frac{S_{L_{i}}}{S_{L_{1}}}=\Delta_{i}, \forall i \in\{1, \ldots, M\}$, such that $S_{L_{i}}$ is directly proportional to $\alpha_{i}^{2}$ in this regime. Consequently, $S_{L_{i}}$ can be expressed as $S_{L_{i}}=\frac{\alpha_{i}^{2}}{\sum_{i=1}^{M} \alpha_{i}^{2}} \sum_{i=1}^{M} S_{L_{i}}$ and $g_{T}\left(S_{T}\right)$, which is utilized in our CFA of (11), can be re-expressed as $g_{T}\left(S_{T}\right)=-e^{-\left(\frac{\mathcal{C} \ln 2}{2 T}+\frac{1}{2}+\ln (2)\right)}$. In turn, the latter further simplifies to

$$
\begin{aligned}
g_{T}\left(S_{T}\right) & \stackrel{0}{\sim}-\frac{1}{2} e^{-\left(\frac{\mathcal{C} \ln (2)}{T}\right)} e^{-\frac{1}{2}\left(1-\frac{\mathcal{C} \ln (2)}{T}\right)} \\
& \stackrel{0}{\sim}-\frac{1}{2}\left(1-\frac{\mathcal{C} \ln (2)}{T}\right) e^{\left(1-\frac{\mathcal{c} \ln (2)}{T}\right)}
\end{aligned}
$$

since $e^{x} \stackrel{0}{\sim} 1+x$ and, hence, $W_{0}\left(g_{T}\left(S_{T}\right)\right)$ $-\frac{1}{2}\left(1-\frac{\mathcal{C} \ln (2)}{T}\right) . \quad$ Similarly, $\quad W_{0}\left(g_{L}\left(S_{L_{i}}\right)\right)$ $-\frac{1}{2}\left(1-\frac{\alpha_{i}^{2} \mathcal{C} \ln 2}{L \sum_{i=1}^{M} \alpha_{i}^{2}}\right) \quad$ and $\quad x_{i} \quad \stackrel{0}{\sim} \frac{L \sum_{i=1}^{M} \alpha_{i}^{2}-\alpha_{i}^{2} \mathcal{C} \ln (2)}{L \sum_{i=1}^{M} \alpha_{i}^{2}-\alpha_{1}^{2} \mathcal{C} \ln (2)}$. By inserting the approximations of $W_{0}\left(g_{T}^{1}\left(S_{T}\right)\right)$ and $W_{0}\left(g_{L}\left(S_{L_{i}}\right)\right)$ into $\bar{g}$ and $\bar{d}_{i}$, respectively, in addition with $x_{i}$ into (11), we obtain upon simplification that

$$
\begin{aligned}
\tilde{f}_{l}^{-1}(\mathcal{C}) \stackrel{0}{\sim} & \frac{2 \mathcal{C} \ln (2)\left(L \sum_{i=1}^{M} \alpha_{i}^{2}+\alpha_{1}^{2} T\right)}{2 \kappa L \sum_{i=1}^{M} \alpha_{i}^{2}(T-\mathcal{C} \ln (2))\left(\sum_{i=1}^{M} \alpha_{i}^{2}+\alpha_{1}^{2} \beta / \kappa\right)} \\
\stackrel{0}{\sim} & \frac{\mathcal{C} \ln (2)\left(L \sum_{i=1}^{M} \alpha_{i}^{2}+\alpha_{1}^{2} T\right)}{T L \sum_{i=1}^{M} \alpha_{i}^{2}\left(\kappa \sum_{i=1}^{M} \alpha_{i}^{2}+\alpha_{1}^{2} \beta\right)}
\end{aligned}
$$

when $\mathcal{C} \rightarrow 0$. Note that $\kappa=L / T, \beta=1$ and $\kappa=1, \beta=$ $T / L$, in the uplink and downlink channels, respectively. Hence we can simplify (39) and express it in the generic form of (20).

\section{Proof of Proposition IV.2}

At high-SE, it can be easily proved that

$$
\begin{aligned}
& u_{i} \stackrel{\infty}{\approx} \frac{\kappa \sum_{k=1}^{M} \Delta_{k} x_{k}^{\infty}-\beta \Delta_{i} x_{i}^{\infty}}{\kappa \sum_{k=1}^{M} \Delta_{k} x_{k}^{\infty}}, i \in\{1, \ldots, M\}, \\
& \frac{d_{0} \sqrt{\gamma}}{\beta}=\stackrel{\infty}{\kappa \sum_{k=1}^{M} \alpha_{k}^{2} x_{k}^{\infty}-\alpha_{1}^{2} \beta},
\end{aligned}
$$

and the ratio of $\frac{u_{i}}{u_{1}}$, i.e. $x_{i}^{\infty}$, converges to a fixed value which can be obtained by solving a series of equation given by

$$
x_{i}^{\infty}=\frac{u_{i}}{u_{1}}=\frac{\kappa \sum_{k=1}^{M} \Delta_{k} x_{k}^{\infty}-\beta \Delta_{i} x_{i}^{\infty}}{\kappa \sum_{k=1}^{M} \Delta_{k} x_{k}^{\infty}-\beta}, i \in\{1, \ldots, M\} .
$$

Note that for the case of $M=2, x_{1}^{\infty}=1$ and $x_{2}^{\infty}=$ $\frac{-U+\sqrt{U^{2}+4 \kappa^{2} \Delta_{2}}}{2 \kappa \Delta_{2}}$, where $U=(\kappa-\beta)\left(1-\Delta_{2}\right)$. A useful observation is that by utilizing the approximation of $\frac{d_{0} \sqrt{\gamma}}{\beta}$ and $x_{i}^{\infty}$ at the high-SE regime, the explicit expression of $S_{L_{i}}$ can be expressed as from (5) as

$$
\begin{aligned}
S_{L_{i}}^{\infty}=\frac{L}{2} & {\left[-1+\frac{\kappa \sum_{k=1}^{M} \Delta_{k} x_{k}^{\infty}-\beta}{\kappa \sum_{k=1}^{M} \Delta_{k} x_{k}^{\infty}-\beta\left(1-\Delta_{i}\right)}\right.} \\
& \left.+2 \ln \frac{\kappa \sum_{k=1}^{M} \Delta_{k} x_{k}^{\infty}-\beta\left(1-\Delta_{i}\right)}{\kappa \sum_{k=1}^{M} \Delta_{k} x_{k}^{\infty}-\beta}\right] .
\end{aligned}
$$

Thus, we can obtain $\sum_{i=1}^{M} S_{L_{i}}^{\infty}$ with (42) and $S_{T}^{\infty}=\mathcal{C} \ln (2)-$ $\sum_{i=1}^{M} S_{L_{i}}^{\infty}$ according to (4), which is also equivalent to

$$
S_{T}^{\infty}=T\left(-\frac{1}{2}-\ln (2)+\frac{1}{1+\bar{g}}+\ln (1+\bar{g})\right) .
$$

Moreover, $\bar{g}=2 g+1$ and $\bar{g}=-\left(1+\frac{1}{W_{0}\left(Q_{0}\right)}\right)$, where $Q_{0}=$ $-\exp \left(-\left(\frac{S_{T}^{\infty}}{T}+\frac{1}{2}+\ln (2)\right)\right)$, hence, we can express $g$ as

$$
g=-\left(1+\frac{1}{2 W_{0}\left(Q_{0}\right)}\right) \text {. }
$$

In addition, note that $g$ can also be defined as $g=$ $\kappa \gamma u_{1} \sum_{i=1}^{M} \alpha_{i}^{2} x_{i}$ which is equivalent to

$$
g=\kappa \gamma\left[\sum_{i=1}^{M} \frac{\alpha_{i}^{2}\left(\kappa \sum_{k=1}^{M} \Delta_{k} x_{k}^{\infty}-\beta\right)}{\kappa \sum_{k=1}^{M} \Delta_{k} x_{k}^{\infty}-\beta\left(1-\Delta_{i}\right)}\right]
$$

at high SE. Consequently, we obtain the closed-form approximation of the inverse function, $f^{-1}(\mathcal{C})$, in the high-SE regime when $M L>T$ in (21) by equating (44) to (45).

D. Proof of Proposition IV.3

Relying on (22) and knowing that $\frac{d_{0}}{\beta \sqrt{\gamma}}=\frac{1}{\beta} \sqrt{\frac{1}{\gamma} \sum_{i=1}^{M} v_{i}}$, $g+1=\beta \sqrt{\frac{\gamma}{\sum_{i=1}^{M} v_{i}}}, d_{1}=\alpha_{1}^{2} \sqrt{\gamma \sum_{i=1}^{M} v_{i}}$ as well as $d_{i}=$ $\Delta_{i} d_{1}$, where $v_{i}=1 / \alpha_{i}^{2}$, the asymptotic approximation of the capacity per unit bandwidth can be expressed as

$\mathcal{C} \ln (2) \approx$

$$
\begin{aligned}
& \frac{L}{2}\left(-M+\frac{\sum_{i=1}^{M} \frac{1}{\Delta}}{\alpha_{1}^{2} \sqrt{\gamma \sum_{i=1}^{M} v_{i}}}+2 \ln \left(\prod_{i=1}^{M} \Delta_{i}\right)+2 M \ln \left(\alpha_{1}^{2} \sqrt{\gamma \sum_{i=1}^{M} v_{i}}\right)\right) \\
& +\frac{T}{2}\left(-1+\frac{1}{\beta} \sqrt{\frac{\sum_{i=1}^{M} v_{i}}{\gamma}+}+2 \ln \left(\beta \sqrt{\frac{\gamma}{\sum_{i=1}^{M} v_{i}}}\right)\right),
\end{aligned}
$$

which can be further simplified as

$$
\begin{aligned}
A_{1}=\frac{L \sum_{i=1}^{M} v_{i}}{\sqrt{\gamma}}+\frac{T}{\beta} & \sqrt{\frac{\sum_{i=1}^{M} v_{i}}{\gamma}}+2 M L \ln \left(\alpha_{1}^{2} \sqrt{\gamma \sum_{i=1}^{M} v_{i}}\right) \\
& +2 T \ln \left(\beta \sqrt{\frac{\gamma}{\sum_{i=1}^{M} v_{i}}}\right)
\end{aligned}
$$

where $A_{1} \approx 2 \mathcal{C} \ln (2)+L\left(M-2 \ln \prod_{i=1}^{M} \Delta_{i}\right)+T$. Since in the uplink and downlink scenarios, $T / L=M$, equation (47) can be re-expressed as

$$
\frac{A_{1}}{2 T}-\ln \left(\alpha_{1}^{2} \beta\right)=\frac{A_{2}}{\sqrt{\gamma}}+\ln (\gamma)
$$

where $A_{2}=\frac{\kappa+1}{2 \beta} \sqrt{\sum_{i=1}^{M} v_{i}}$. Hence, by expressing $\gamma$ in terms of a dummy variable $D$ such that $\gamma=\left(A_{2} / 2\right)^{2} D$, equation (48) simplifies to $-\frac{A_{1}}{4 T}+\frac{1}{2} \ln \left(\alpha_{1}^{2} \beta\right)+\ln \left(\frac{A_{2}}{2}\right)=-\frac{1}{\sqrt{D}}+$ $\ln \frac{1}{\sqrt{D}}$ such that 


$$
-\exp \left(-\frac{A_{1}}{4 T}+\frac{1}{2} \ln \left(\alpha_{1}^{2} \beta\right)+\ln \left(\frac{A_{2}}{2}\right)\right)=-\frac{1}{\sqrt{D}} \exp ^{-\frac{1}{\sqrt{D}}} .
$$

Using the real branch of the Lambert W function [29], equation (49) can be reformulated as

$$
-\frac{1}{\sqrt{D}}=W_{0}\left(Q_{1}\right)
$$

where $Q_{1}$ is expressed in equation (24). Hence, the highSE approximation of the inverse function, $f^{-1}(\mathcal{C})$, used in expressing the DMIMO EE-SE trade-off is obtained when $M L=T$ in (23) by replacing the dummy variable $D$ with $\gamma \simeq \tilde{f}_{h}^{-1}(\mathcal{C})$.

\section{E. Proof of Proposition IV.4}

Since the high-SE approximation of the unique positive real root of the $(M+1)^{t h}$ degree polynomial in (3) is such that $\frac{\beta \sqrt{\gamma}}{d_{0}}=1+g=\frac{\beta}{\beta-M \kappa}$, it implies that the high-SE approximation of $S_{T}$ in (5), i.e. $S_{T}^{\infty}$, is independent of $\gamma$ and can be expressed as

$$
\begin{aligned}
S_{T}^{\infty} & =\frac{T}{2}\left(-1+\frac{\beta-M \kappa}{\beta}+2 \ln \left(\frac{\beta}{\beta-M \kappa}\right)\right) \\
& =-\frac{M L}{2}-T \ln \left(1-\frac{M \kappa}{\beta}\right) .
\end{aligned}
$$

Furthermore, we know from (4) that sum of $S_{L_{i}}$ at high-SE can be expressed in terms of the capacity per unit bandwidth $\mathcal{C}$ which simplifies to

$$
\begin{aligned}
\sum_{i=1}^{M} S_{L_{i}}^{\infty} & =\mathcal{C} \ln (2)-S_{T}^{\infty} \\
& =\frac{L}{2}\left(-M+\frac{1}{d_{1}} \sum_{i=1}^{M} \frac{1}{\Delta_{i}}+2 \ln \left(\Delta_{i}\right)+2 M \ln \left(d_{1}\right)\right)
\end{aligned}
$$

Let us define $\tilde{d}_{1}=2 M d_{1}+1$, hence equation (52) can be re-expressed such that

$$
\begin{aligned}
Q_{2} & =-\left(\frac{\mathcal{C} \ln (2)-S_{T}^{\infty}}{M p}+\frac{1}{2}-\frac{1}{M} \ln \left(\prod_{i=1}^{M} \Delta_{i}\right)+\ln (2 M)-\ln \left(\sum_{i=1}^{M} \frac{1}{\Delta_{i}}\right)\right) \\
& =\quad-\frac{1}{\tilde{d}_{1}-1} \sum_{i=1}^{M} \frac{1}{\Delta_{i}}+\ln \left(\frac{1}{\tilde{d}_{1}-1} \sum_{i=1}^{M} \frac{1}{\Delta_{i}}\right) .
\end{aligned}
$$

Using the real branch of the Lambert W function [29], equation (53) can be reformulated as

$$
\tilde{d}_{1}=1-\frac{\sum_{i=1}^{M} \frac{1}{\Delta_{i}}}{W_{0}\left(Q_{2}\right)} .
$$

Since $\tilde{d}_{1}=2 M d_{1}+1$ and $d_{1}=d_{0} \alpha_{1}^{2} \sqrt{\gamma}$, by substituting $d_{0}=\sqrt{\gamma}(\beta-M \kappa)$ in $d_{1}$, then $d_{1}$ in $\tilde{d}_{1}$ and the later in (54), it can be easily shown that

$$
\gamma \simeq \tilde{f}_{h}^{-1}(\mathcal{C})=-\frac{\sum_{i=1}^{M} v_{i}}{2 M(\beta-M \kappa) W_{0}\left(Q_{2}\right)}
$$

for the scenario where $M L<T$ in the DMIMO system.

\section{REFERENCES}

[1] H. Kwon and T. Birdsall, "Channel Capacity in Bits per Joule," IEEE J. Ocean. Eng., vol. 11, no. 1, pp. 97-99, Jan. 1986.

[2] S. Verdu, "Spectral Efficiency in the Wideband Regime," IEEE Trans. Inf. Theory, vol. 48, no. 6, pp. 1319-1343, Jun. 2002.

[3] A. Lozano, A. M. Tulino, and S. Verdu, "Multiple-Antenna Capacity in the Low-Power Regime," IEEE Trans. Inf. Theory, vol. 49, no. 10, pp. 2527-2544, 2003.

[4] G. Caire, D. Tuninetti, and S. Verdu, "Suboptimality of TDMA in the Low-Power Regime," IEEE Trans. Inf. Theory, vol. 50, no. 4, pp. 608620, 2004.

[5] O. Oyman and S. Sandhu, "A Shannon-Theoretic Perspective on Fading Multihop Networks," in Proc. 40th Annual Conf. Information Sciences and Systems, 2006, pp. 525-530.
[6] O. Arnold, F. Richter, G. Fettweis, and O. Blume, "Power Consumption Modeling of Different Base Station Types in Heterogeneous Cellular Networks," in Proc. Future Network and Mobile Summit, Florence, Italy, Jun. 2010.

[7] A. J. Fehske, P. Marsch, and G. P. Fettweis, "Bit per Joule Efficiency of Cooperating Base Stations in Cellular Networks," in Proc. IEEE Globecom, Miami, USA, Dec. 2010.

[8] G. Auer et al., "How Much Energy is Needed to Run a Wireless Network?" IEEE Wireless Commun. Mag., vol. 18, no. 5, pp. 40-49, 2011.

[9] S. Tombaz et al, "Impact of Backhauling Power Consumption on the Deployment of Heterogeneous Mobile Networks," in Proc. IEEE Global Telecommunications Conf. (GLOBECOM 2011), 2011, pp. 1-5.

[10] G. Miao, N. Himayat, and G. Y. Li, "Energy-Efficient Link Adaptation in Frequency-Selective Channels," IEEE Transactions on Communications, vol. 58, no. 2, pp. 545-554, Feb. 2010.

[11] F. Héliot, O. Onireti, and M. A. Imran, "An Accurate Closed-Form Approximation of the Energy Efficiency-Spectral Efficiency TradeOff over the MIMO Rayleigh Fading Channel," in Proc. IEEE Int Communications Workshops (ICC) Conf, 2011, pp. 1-6.

[12] F. Héliot, M. A. Imran, and R. Tafazolli, "On the Energy EfficiencySpectral Efficiency Trade-Off over the MIMO Rayleigh Fading Channel," IEEE Trans. Commun., vol. 60, no. 5, pp. 1345-1356, May 2012.

[13] O. Onireti, F. Héliot, and M. Imran, "On the Energy Efficiency-Spectral Efficiency Trade-Off in the Uplink of CoMP System," IEEE Trans. Wireless Commun., vol. 11, no. 2, pp. 556-561, Feb. 2012.

[14] A. A. M. Saleh, A. Rustako, and R. Roman, "Distributed Antennas for Indoor Radio Communications," IEEE Transactions on Communications, vol. 35, no. 12, pp. 1245-1251, Dec. 1987.

[15] W. Roh and A. Paulraj, "MIMO Channel Capacity for the Distributed Antenna," in Proc. VTC 2002-Fall Vehicular Technology Conf. 2002 IEEE 56th, vol. 2, 2002, pp. 706-709.

[16] _ - "Outage Performance of the Distributed Antenna Systems in a Composite Fading Channel," in Proc. IEEE 56th Vehicular Technology Conf. (VTC Fall), vol. 3, 2002, pp. 1520-1524.

[17] D. Castanheira and A. Gameiro, "Distributed Antenna System Capacity Scaling," IEEE Wireless Commun. Mag., vol. 17, no. 3, pp. 68-75, 2010.

[18] X.-H. You, D.-M. Wang, B. Sheng, X.-Q. Gao, X.-S. Zhao, and M. Chen, "Cooperative Distributed Antenna Systems for Mobile Communications," IEEE Wireless Commun. Mag., vol. 17, no. 3, pp. 35-43, 2010.

[19] L. Dai, " A Comparative Study on Uplink Sum Capacity with CoLocated and Distributed Antenna," IEEE J. Sel. Areas Commun., vol. 29, no. 6, pp. 1200-1213, June 2011.

[20] D. Wang, X. You, J. Wang, Y. Wang, and X. Hou, "Spectral Efficiency of Distributed MIMO Cellular Systems in a Composite Fading Channel," in Proc. IEEE Int. Conf. Communications ICC '08, 2008, pp. 1259-1264.

[21] F. Héliot, R. Hoshyar, and R. Tafazolli, "An Accurate Closed-Form Approximation of the Distributed MIMO Outage Probability," IEEE Trans. Wireless Commun., vol. 10, no. 1, pp. 5-11, 2011.

[22] F. Héliot, M. A. Imran, and R. Tafazolli, "Energy Efficiency Analysis of Idealized Coordinated Multi-Point Communication System," in Proc. IEEE 73rd Vehicular Technology Conf. (VTC Spring), 2011, pp. 1-5.

[23] S. Lee, S. Moon, J. Kim, and I. Lee, "Capacity Analysis of Distributed Antenna Systems in a Composite Fading Channel," IEEE Trans. Wireless Commun., vol. 11, no. 3, pp. 1076-1086, Mar. 2012.

[24] C. He, B. Sheng, D. Wang, P. Zhu, and X. You, "Energy Efficiency Comparison between Distributed MIMO and Co-Located MIMO Systems," Int. J. Commun. Syst., pp. 1-14, 2012.

[25] C. He, B. Sheng, P. Zhu, and X. You, "Energy Efficiency and Spectral Efficiency Tradeoff in Downlink Distributed Antenna Systems," IEEE Wireless Commun. Lett., vol. 1, no. 3, pp. 153-156, June 2012.

[26] N. C. Beaulieu and F. Rajwani, "Highly Accurate Simple Closed-Form Approximations to Lognormal Sum Distributions and Densities," IEEE Commun. Lett., vol. 8, no. 12, pp. 709-711, 2004.

[27] M. Dohler and H. Aghvami, "On the Approximation of MIMO Capacity," IEEE Trans. Wireless Commun., vol. 4, no. 1, pp. 30-34, 2005.

[28] O. Onireti, F. Héliot, and M. A. Imran, "On the Energy EfficiencySpectral Efficiency Trade-Off of the 2BS-DMIMO System," in Proc. IEEE 76th Vehicular Technology Conf. (VTC Fall), 2012.

[29] R. M. Corless, G. H. Gonnet, D. E. G. Hare, D. J. Jeffrey, and D. E. Knuth, "On the LambertW Function," Adv. Comput. Math., vol. 5, pp. 329-359, 1996. 


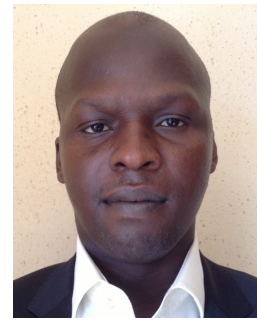

Oluwakayode Onireti (S'11-M'13) received his B.Eng. degree in Elecrical Engineering from University of Ilorin, Nigeria, in 2005. The M.Sc. in Mobile \& Satellite Communications and Ph.D. degrees in Electronics Engineering from University of Surrey, UK, in 2009 and 2012, respectively. He secured a first class grade in his B.Eng. and a distinction in his M.Sc. degree. He is currently a research fellow at the Centre for Communication Systems Research (CCSR) at the University of Surrey, UK. He has been actively involved in European Commission funded projects such as ROCKET and EARTH. His main research interests include energy efficiency, MIMO and cooperative communications

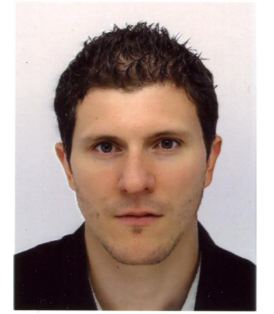

Fabien Héliot (S'05-M'07) received the M.Sc. degree in Telecommunications from the Institut Supérieur de l'Electronique et du Numérique (ISEN), Toulon, France, and the Ph.D. degree in Mobile Telecommunications from King's College London, in 2002 and 2006, respectively. He is currently a research fellow at the Centre for Communication Systems Research (CCSR) of the University of Surrey. He has been actively involved in European Commission funded projects such as FIREWORKS, ROCKET, SMART-Net projects and more recently in the award-winning EARTH project. He is currently involved in the LexNet project, a project investigating human exposure to electromagnetic fields induced by wireless telecommunication networks. His main research interests are energy efficiency, cooperative communication, MIMO, and radio resource management. He received an Exemplary Reviewer Award from IEEE COMMUNICATIONS LETTERS in 2011.

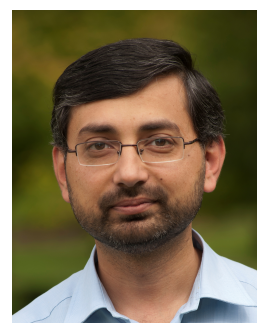

Muhammad Ali Imran (M'03-SM'12) received his B.Sc. degree in Electrical Engineering from University of Engineering and Technology Lahore, Pakistan, in 1999, and the M.Sc. and Ph.D. degrees from Imperial College London, UK, in 2002 and 2007, respectively. He secured first rank in his $\mathrm{BSc}$ and a distinction in his MSc degree along with an award of excellence in recognition of his academic achievements, conferred by the President of Pakistan. He is currently a Reader in the Centre for Communication Systems Research (CCSR) at the University of Surrey, UK. He has been actively involved in European Commission funded research projects ROCKET, EARTH, LexNet and iJoin; Mobile VCE funded project on fundamental capacity limits; EPSRC funded India-UK ATC and REDUCE projects. He is chairing the work area on the air interface design under the $5 \mathrm{G}$ innovation centre. His main research interests include the analysis and modelling of the physical layer, optimization for the energy efficient wireless communication networks and the evaluation of the fundamental capacity limits of wireless networks. 